\title{
Application of a buoyancy-modified $k-\omega$ SST turbulence model to simulate wave run-up around a monopile subjected to regular waves using OpenFOAM ${ }^{\circledR}$
}

\author{
Brecht Devolder ${ }^{a, b, *}$, Pieter Rauwoens ${ }^{b}$, Peter Troch $^{a}$ \\ a Ghent University, Department of Civil Engineering, Technologiepark 904, 9052 Ghent, Belgium \\ b KU Leuven, Department of Civil Engineering, Technology Cluster Construction, Zeedijk 101, 8400 Ostend, \\ Belgium
}

* Corresponding author at: Ghent University, Department of Civil Engineering, Technologiepark 904, 9052 Ghent, Belgium.

E-mail addresses: Brecht.Devolder@UGent.be (B. Devolder), pieter.rauwoens@kuleuven.be (P. Rauwoens), Peter.Troch@UGent.be (P.Troch).

\section{ABSTRACT}

The objective of the present work is to investigate wave run-up around a monopile subjected to regular waves inside a numerical wave flume using the Computational Fluid Dynamics (CFD) toolbox OpenFOAM $^{\circledR}$. Reynolds-Averaged Navier-Stokes (RANS) turbulence modelling is performed by applying the $k-\omega S S T$ model. Boundary conditions for wave generation and absorption are adopted from the IHFOAM toolbox. Simulations of propagating water waves show sometimes excessive wave damping (i.e. a significant decrease in wave height over the length of the numerical wave flume) based on RANS turbulence modelling. This anomaly is prevented by implementing a buoyancy term in the turbulent kinetic energy equation. The additional term suppresses the turbulence level at the interface between water and air. The proposed buoyancy-modified $k-\omega S S T$ turbulence model results in an overall stable wave propagation model without significant wave damping over the length of the flume. Firstly, the necessity of a buoyancy-modified $k-\omega$ SST turbulence model is demonstrated for the case of propagating water waves in an empty wave flume. Secondly, numerical results of wave run-up around a monopile under regular waves using the buoyancy-modified $k-\omega S S T$ turbulence model are validated by using experimental data measured in a wave flume by De Vos et al. (2007). Furthermore, timedependent high spatial resolutions of the numerically obtained wave run-up around the monopile are presented. These results are in line with the experimental data and available analytical formulations.

Keywords:

CFD; OpenFOAM ${ }^{\circledast} /$ IHFOAM; Buoyancy-modified turbulence model; Monopile; Wave run-up

Postprint accepted for publication in Coastal Engineering, July 2017, vol. 125, pp. 81-94. 


\section{Introduction}

Numerous offshore wind farms have already been installed or are under construction. Wind turbines are mounted on large foundations in the seabed, such as vertical cylinders, called monopiles. The design of such a monopile is mainly dependent on the total force acting on it. However, some smaller pieces are attached to that monopile (e.g. boat landing facility, J-tube, ladder, platform and door). In order to design these smaller parts, wave run-up around the monopile caused by incident waves should be assessed accurately. Therefore, both experimental and numerical research have already been conducted.

Experimental research has been performed in order to define the wave run-up pattern around a monopile. For example, De Vos et al. (2007) described small scale model tests in which wave run-up was measured around a monopile placed in relatively deep water conditions using different regular and irregular wave trains. Moreover, analytical formulations are proposed to determine the maximum wave run-up for both regular and irregular waves. Kazeminezhad and Etemad-Shahidi (2015) have recently re-analysed several datasets and presented alternative formulations in which pre-calculation of the wave kinematics is not necessary to assess the maximum wave run-up.

Numerical modelling of wave run-up around a monopile is also reported in literature. Christensen et al. (2005) described a study of the forces acting on a monopile caused by extreme waves propagating over a sloping bed. Numerical results were compared with analytical solutions and experiments and a good agreement was found. A numerical study with a 3-D ComFLOW model performed by Peng et al. (2012) reproduced experimental data measured by De Vos et al. (2007). A grid sensitivity study showed that a minimum grid size of $D / 10$ was needed in the zones of interest (i.e. around the still water level and near the monopile with diameter $D$ ) to obtain a grid independent solution. The Courant-FriedrichsLewy (CFL) condition controlled the time step which may not exceed $T / 100$ for accuracy purposes (where $T$ is the wave period). The paper presented only absolute values of wave run-up and no comparisons with regard to time series were provided. The authors also mentioned that for large wave run-up, the numerical model slightly underestimates the measured run-up. Lara et al. (2013) presented a numerical simulation of a pile group subjected to waves using the IHFOAM toolbox. Only numerical results were presented which indicated that IHFOAM is a capable toolbox for analysing wave run-up around and wave-induced forces on offshore piles. Ransley et al. (2013) compared numerical results with experimental data for extreme wave impacts on a fixed truncated circular cylinder. The numerical solution was obtained without turbulence modelling but the authors expect that it plays an important role in (extreme) wave-structure interaction. El Safti et al. (2014) presented a hybrid 2D-3D CFD model to investigate wave forces on piled structures. In this study, turbulent effects were incorporated by using a one-equation eddy-viscosity Sub-Grid Scale (SGS) Large Eddy Simulation (LES) model. The authors' motivation to use LES was to include the effects of air compressibility during breaking wave impact on structures. Paulsen et al. (2014) analysed strong nonlinear forces caused by steep or breaking waves and ringing loads due to steep nonlinear waves. Turbulence modelling was excluded because the forces acting on the monopile were mainly inertia dominated. A fair agreement was found between numerical and experimental data. Chen et al. (2014) investigated nonlinear wave interactions with offshore structures for different wave conditions. They concluded that OpenFOAM is suitable for accurate modelling of nonlinear wave interactions with monopiles. The time step was initially $0.01 \mathrm{~s}$ and changed automatically to satisfy a maximum Courant number of 0.5 . Grid convergence was reached for a horizontal and vertical resolution of respectively $L / 70$ and $H / 8$ (with a refinement factor of 2 around the still water level and the monopile; where $L$ is the wave length and $H$ is the wave height).

More recently, Kamath et al. (2015a, 2015b) reported CFD results of wave interaction with multiple vertical cylinders. They performed simulations using a $k-\omega$ turbulence model and observed unphysical 
wave damping based on RANS turbulence modelling. Therefore, both eddy viscosity limiters and free surface turbulence damping at the interface were applied. This unphysical wave damping caused by RANS turbulence modelling is not only observed during CFD simulations of monopiles. Several other authors also reported wave damping when using CFD for wave modelling: Mayer and Madsen (2000), Jacobsen et al. (2012), Vanneste and Troch (2015) and Elhanafi et al. (n.d.).

In general, the majority of literature presents wave-induced forces acting on a monopile rather than wave run-up phenomena. Therefore, turbulence modelling was omitted and no indication of the influence of turbulence on wave run-up was given. However, some authors reported the necessity of using a turbulence model. For example Higuera et al. (2013a, 2013b) applied both $k-\varepsilon$ and $k-\omega$ SST turbulence models since they are widely used. Furthermore, turbulence modelling is needed in the case of significant vortex shedding or when wave breaking occurs around the monopile due to even steeper waves. This can happen when irregular waves are generated, then energy is transferred between the different frequencies increasing the wave height at a particular time instant and at a certain location. If that location is close to the monopile, waves can break and prominent vortex shedding can occur. This paper will tackle the implementation of a suitable turbulence model in order to simulate properly wave propagation in a numerical wave flume and wave run-up around a monopile. In general, non-breaking waves induce a very low level of turbulence. However, when the waveinduced flow encounters an object, a monopile in this case, the flow is disturbed and becomes turbulent. For the waves studied in this research, the Reynolds (Re) number and Keulegan-Carpenter (KC) number vary from $4.65 \cdot 10^{4}$ to $5.84 \cdot 10^{4}$ and from 4.26 to 5.17 respectively. According to Sumer and Fredsøe (1997), a pair of asymmetric vortices will develop resulting in a turbulent flow around the monopile. Moreover, even if the KC numbers are small for the waves studied, the boundary layer around the monopile may be turbulent.

We present a study of wave run-up around a monopile subjected to regular waves using the Computational Fluid Dynamics (CFD) toolbox OpenFOAM ${ }^{\circledR}$ (2013). Reynolds-Averaged Navier-Stokes (RANS) turbulence modelling is performed by applying the $k-\omega$ SST model. Sometimes, this RANS approach causes excessive wave damping (i.e. a significant decrease in wave height over the length of the numerical wave flume). Therefore, a buoyancy term is implemented in the turbulent kinetic energy (TKE) equation of the $k-\omega S S T$ model. The idea of adding a buoyancy term is taken from Van Maele and Merci (2006) who modify the $k-\varepsilon$ model to simulate buoyant plumes. These fire-induced flows are characterised by continuous density variations. For water waves, the density is discontinuous at the free water surface resulting in an infinite density gradient. However, when a Volume of Fluid (VoF) method is applied for wave modelling, the density gradient is smeared out over several cells leading to a continuous change in density around the air-water interface. Consequently, the change in density around the interface between water and air is similar to the change in density observed in fire flows. As a result of implementing a buoyancy term, an overall stable wave propagation model without significant wave damping over the length of the flume is obtained. Numerical simulations are performed and compared with two different sets of wave parameters described in the laboratory study of De Vos et al. (2007).

The remainder of this paper is organised as follows. Firstly, in Section 2, the previous experimental study by De Vos et al. (2007) is introduced. In Section 3, the governing equations for the numerical model are presented, followed by a description of the computational domain, the boundary conditions applied and the solver settings. Subsequently in Sections 4 and 5, the numerical model is used to perform several simulations while in Section 6 the obtained results are discussed in detail. Finally, the conclusions and future work are drawn in Section 7. 


\section{Previous experimental study}

In this research, data is reused from a laboratory study by De Vos et al. (2007) conducted in a wave flume at Aalborg University, Denmark. The flume has a length of $30 \mathrm{~m}$, a width of $1.5 \mathrm{~m}$ and a height of $1 \mathrm{~m}$. The pile diameter was $0.12 \mathrm{~m}$ whereas a constant water depth of $0.50 \mathrm{~m}$ was maintained during regular wave tests. The offshore slope was held constant at 1:100 and will be neglected in the present numerical study because shoaling effects are negligible.

A definition sketch of the wave gauges' position to measure wave run-up around the monopile is given in Figure 1. Herein, nine wave gauges are installed $2 \mathrm{~mm}$ away from the monopile's surface. The position of the wave gauges is an important parameter to determine wave run-up. Therefore, the position of the numerical wave gauges is the same as the ones installed in the experimental flume. The nine wave gauges are characterised by their angle with respect to the incoming waves of respectively $0^{\circ}, 22.5^{\circ}, 45^{\circ}, 67.5^{\circ}, 90^{\circ}, 112.5^{\circ}, 135^{\circ}, 157.5^{\circ}$ and $180^{\circ}$, as depicted in Figure 1.

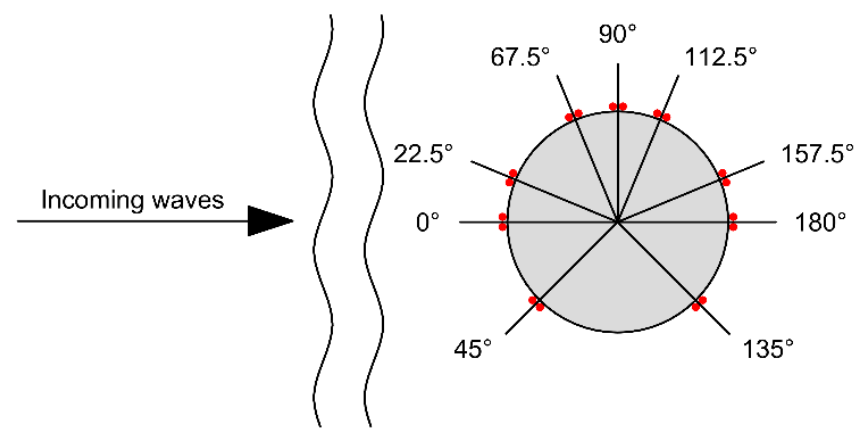

Figure 1: Position of the nine wave gauges (two dots per gauge) around the monopile where each wave gauge is characterised by its angle with respect to the direction of the incoming waves. The wave gauges are installed $2 \mathrm{~mm}$ away from the monopile's surface (adapted from De Vos et al., 2007).

\section{Numerical model}

The numerical simulations presented are achieved using OpenFOAM, version 2.2.2. Firstly, the flow equations are introduced with emphasis on the turbulence model. Subsequently, the computational domain is presented together with the grid characteristics. The last two parts of this section are dedicated to explain the different boundary conditions and solver settings.

\subsection{Flow equations}

The numerical model uses the incompressible RANS equations to express the motion of a fluid consisting of a mass conservation equation (1) and a momentum conservation equation (2) written in Einstein summation notation as:

$$
\begin{gathered}
\frac{\partial u_{i}}{\partial x_{i}}=0 \\
\frac{\partial \rho u_{i}}{\partial t}+\frac{\partial \rho u_{j} u_{i}}{\partial x_{j}}-\frac{\partial}{\partial x_{j}}\left[\mu_{e f f} \frac{\partial u_{i}}{\partial x_{j}}\right]=-\frac{\partial p^{*}}{\partial x_{i}}+F_{b, i}+f_{\sigma, i}
\end{gathered}
$$

in which $u_{i}(i=x, y, z)$ are the Cartesian components of the fluid velocity, $\rho$ is the fluid density, $\mu_{\text {eff }}$ is the effective dynamic viscosity, $p^{*}$ is the pressure in excess of the hydrostatic, $F_{b}$ is an external body force (including gravity) and $f_{\sigma}$ is the surface tension tensor term which will be neglected. Note that the mean values for the variables considered are written in terms of Favre-averaging (density weighted) due to the varying density.

The interface between water and air is obtained by the Volume of Fluid (VoF) method (Hirt and Nichols, 1981). The method is based on a volume fraction $\alpha$ which is 0 for a completely dry cell and 1 for a 
completely wet cell and in between 0 and 1 for an interface cell containing both water and air. The volume fraction is solved by an advection equation (3):

$$
\frac{\partial \alpha}{\partial t}+\frac{\partial u_{i} \alpha}{\partial x_{i}}+\frac{\partial u_{c, i} \alpha(1-\alpha)}{\partial x_{i}}=0
$$

The last term on the left-hand side is an artificial compression term where $u_{c, i}=\min \left[c_{\alpha}\left|u_{i}\right|, \max \left(\left|u_{i}\right|\right)\right]$. In the present study, the default value of $c_{\alpha}$ equal to 1 is applied. If a larger value is used, the compression of the interface increases, leading to larger detrimental velocity gradients around that interface.

The density of the fluid $\rho$ within a computational cell is calculated by a weighted value based on the volume fraction $\alpha$. The effective dynamic viscosity $\mu_{\text {eff }}$ is obtained by the sum of a weighted value based on the volume faction $\alpha$ and an additional turbulent dynamic viscosity $\rho v_{t}$ :

$$
\begin{gathered}
\rho=\alpha \rho_{\text {water }}+(1-\alpha) \rho_{\text {air }} \\
\mu_{\text {eff }}=\alpha \mu_{\text {water }}+(1-\alpha) \mu_{\text {air }}+\rho v_{t}
\end{gathered}
$$

In a post processing step, the position of the free water surface is determined by a discrete integration of the volume fraction $\alpha$ over a vertical line (Z-direction) divided in $n$ equal parts:

$$
z_{\text {water level }}=\sum_{i=0}^{n-1} \alpha_{i}\left(z_{i+1}-z_{i}\right)
$$

\subsection{Turbulence modelling}

Turbulent effects are incorporated in the RANS equations (1) and (2) by solving one or more additional transport equations to yield a value for the turbulent kinematic viscosity $v_{t}$. The $k-\omega S S T$ turbulence model is applied in all the simulations presented. $k-\omega S S T$ has shown good results in literature to simulate the flow around circular cylinders and two-phase flows. For example, Rahman et al. (2008) mentioned that the $k$ - $\omega$ SST turbulence model is much more recommendable for high Reynolds numbers in a uniform free stream flow passing a 2D cylinder. Moreover, it has an adequate boundary layer treatment. Brown et al. (2014) evaluated different RANS turbulence models for spilling breakers and concluded that the $k-\omega S S T$ model performs best for wave elevation predictions.

The incompressible $k-\omega$ SST model for a single fluid is a two-equation model (Menter et al., 2003) and is formulated in OpenFOAM as:

$$
\begin{gathered}
\frac{\partial k}{\partial t}+\frac{\partial u_{j} k}{\partial x_{j}}-\frac{\partial}{\partial x_{j}}\left[\left(v+\sigma_{k} v_{t}\right) \frac{\partial k}{\partial x_{j}}\right]=P_{k}-\beta^{*} \omega k \\
\frac{\partial \omega}{\partial t}+\frac{\partial u_{j} \omega}{\partial x_{j}}-\frac{\partial}{\partial x_{j}}\left[\left(v+\sigma_{\omega} v_{t}\right) \frac{\partial \omega}{\partial x_{j}}\right]=\frac{\gamma}{v_{t}} G-\beta \omega^{2}+2\left(1-F_{1}\right) \frac{\sigma_{\omega 2}}{\omega} \frac{\partial k}{\partial x_{j}} \frac{\partial \omega}{\partial x_{j}} \\
P_{k}=\min \left(G, 10 \beta^{*} k \omega\right) \quad G=v_{t} \frac{\partial u_{i}}{\partial x_{j}}\left(\frac{\partial u_{i}}{\partial x_{j}}+\frac{\partial u_{j}}{\partial x_{i}}\right) \quad v_{t}=\frac{a_{1} k}{\max \left(a_{1} \omega, S F_{2}\right)}
\end{gathered}
$$

where $k$ is the turbulent kinetic energy, $P_{k}$ is the production term of $k, v$ is the kinematic viscosity, $v_{t}$ is the turbulent kinematic viscosity, $\omega$ is the specific dissipation rate, $S$ is the mean rate of strain of the flow, $\beta^{*}=0.09, a_{1}=0.31, F_{1}$ and $F_{2}$ are blending functions. The values of $\sigma_{k}, \sigma_{\omega}, \beta$ and $\gamma$ are blended using equation (10) in which $\phi_{1}$ and $\phi_{2}$ are given in Table 1.

$$
\phi=F_{1} \phi_{1}+\left(1-F_{1}\right) \phi_{2}
$$


Table 1: Default values for $\phi_{1}$ and $\phi_{2}$ used in equation (10) to calculate $\sigma_{k}, \sigma_{\omega}, \beta$ and $\gamma$ for the $k-\omega$ SST turbulence model.

\begin{tabular}{c|cccc}
\hline$\phi$ & $\sigma_{k}$ & $\sigma_{\omega}$ & $\beta$ & $\gamma$ \\
\hline$\phi_{1}$ & 0.85034 & 0.5 & 0.075 & 0.5532 \\
\hline$\phi_{2}$ & 1.0 & 0.85616 & 0.0828 & 0.4403 \\
\hline
\end{tabular}

Alternative turbulence models, such as $k-\varepsilon$ and $k-\omega$, were also tested in this study but they caused more wave damping over the length of the wave flume compared to $k-\omega S S T$. Furthermore, application of LES obliges very fine grids in order to resolve $80 \%$ of the turbulence resulting in longer simulation times. Therefore, RANS modelling with the $k-\omega S S T$ model is selected.

However, for high steepness waves, even the original $k-\omega S S T$ model causes significant wave damping. This damping is triggered by an increase in turbulent viscosity around the interface between water and air. This increase is induced by the large production of turbulent kinetic energy, $k$, in that zone. The production of $k$ is linked to the velocity gradient which is large around the interface between water and air due to the large density ratio (1000/1). Two important insights are made according to the original $k-\omega S S T$ model implemented in OpenFOAM, equations (7) and (8):

1. The first one is already reported by Brown et al. (2014). None of the incompressible solvers implemented in OpenFOAM, including two-phase flow, explicitly comprise the density. Only the turbulent kinematic viscosity $v_{t}$ is modelled rather than the dynamic viscosity $\mu_{t}=\rho v_{t}$. In a two-phase flow, the density varies around the interface between water and air which should therefore be included in the turbulence equations.

2. As mentioned in Van Maele and Merci (2006), a buoyancy term is needed in order to take the varying density around the air-water interface into account. The buoyancy term is only included in the turbulent kinetic energy (TKE) equation based on the Standard Gradient Diffusion Hypothesis (SGDH) where the density ratio is neglected. This density ratio is $1000 / 1$ for water and air and could lead to instabilities in the solution method of this study. Moreover, Van Maele and Merci (2006) reported that the influence of buoyancy on the $\varepsilon$-equation (cfr. $\omega$-equation) is negligible if the SGDH is used. However, neglecting buoyancy in the TKEequation is influencing the results significantly. The more advanced Generalized Gradient Diffusion Hypothesis (GGDH) leads to failing simulations during this research due to an instability in the TKE-equation.

Based on those two insights, a modified $k-\omega$ SST model is implemented in OpenFOAM to prevent significant wave damping over the length of the wave flume. Firstly, the density $\rho$ is explicitly implemented in both equations (11) and (12) of the $k$ - $\omega S S T$ turbulence model to take its variability around the air-water interface into account. Secondly, a buoyancy term $G_{b}$ described by the SGDH, equation (13), is added to the TKE-equation (11). The final equations for this buoyancy-modified $k-\omega$ SST turbulence model are formulated as follow:

$$
\begin{gathered}
\frac{\partial \rho k}{\partial t}+\frac{\partial \rho u_{j} k}{\partial x_{j}}-\frac{\partial}{\partial x_{j}}\left[\rho\left(v+\sigma_{k} v_{t}\right) \frac{\partial k}{\partial x_{j}}\right]=\rho P_{k}+G_{b}-\rho \beta^{*} \omega k \\
\frac{\partial \rho \omega}{\partial t}+\frac{\partial \rho u_{j} \omega}{\partial x_{j}}-\frac{\partial}{\partial x_{j}}\left[\rho\left(v+\sigma_{\omega} v_{t}\right) \frac{\partial \omega}{\partial x_{j}}\right] \\
=\frac{\gamma}{v_{t}} \rho G-\rho \beta \omega^{2}+2\left(1-F_{1}\right) \rho \frac{\sigma_{\omega 2}}{\omega} \frac{\partial k}{\partial x_{j}} \frac{\partial \omega}{\partial x_{j}} \\
G_{b}=-\frac{v_{t}}{\sigma_{t}} \frac{\partial \rho}{\partial x_{j}} g_{j}
\end{gathered}
$$


in which the buoyancy term $G_{b}$ is treated implicitly, the scalar $\sigma_{t}=0.85$ and vector $g=[0 ; 0 ;-9.81] \mathrm{m} / \mathrm{s}^{2}$. The value of $\sigma_{t}$ decides how much buoyancy is modelled and is kept constant during all the simulations presented. It is beyond the scope of this research to find the optimal value of $\sigma_{t}$ to return the most accurate results. The purpose of including a buoyancy term in this study is to suppress the turbulence level at the free water surface, i.e. in the zone where a vertical density gradient exists. Because of the implicit treatment of the buoyancy term $G_{b}$ in the TKE-equation, the very large vertical density gradient near the free water surface drives the turbulent viscosity $v_{t}$ to zero. This is demonstrated in Figure 5 and Figure 7 later in this paper. As a result, in case of propagating waves, the model switches to the laminar regime near the free water surface, preventing excessive wave damping. Please note that in the zone of e.g. breaking waves with a more horizontal density gradient, the original turbulence model will be recovered, as $G_{b}=0$.

\subsection{Computational domain}

Because the geometry of the experimental wave flume is symmetrical around its length axis, only one half of the wave flume is modelled in the numerical model. As mentioned in Section 1, a pair of asymmetric vortices will develop but no vortex shedding is expected. Therefore, preliminary simulations are performed without the symmetry plane, by modelling the full domain. As a result, no differences in the wave run-up pattern around the monopile are observed. Moreover, Saghafian et al. (2003) reported small lift forces when the KC number is smaller than 6 for a Reynolds number of 11240 . Furthermore, vortex shedding become prominent if $K C>7$. Consequently, only simulations using a symmetry plane are presented. Figure 2 shows a plan view of the computational domain together with the boundary conditions types which are listed in the next Section 3.4. As mentioned before, the bottom of the numerical domain is flat. The height of the numerical wave flume is $0.80 \mathrm{~m}$ in the Z-direction. The initial condition consists of a water depth of $0.50 \mathrm{~m}$ for which the volume fraction $\alpha$ equal to 1 and on top $0.30 \mathrm{~m}$ air for which $\alpha$ is equal to 0 .

It is important to stress that the length of the numerical flume $(L=20 \mathrm{~m})$ is reduced compared to the length of the experimental flume $(L=30 \mathrm{~m})$. Moreover, the pile was not placed in the centre of the experimental wave flume (in longitudinal direction) whereas in the numerical setup, the pile is placed centrally in the computational domain. The length of the numerical wave flume is chosen to allow a few wave lengths inside the flume to assess the quality of propagating water waves using the buoyancy-modified $k-\omega S S T$ turbulence model.

After discretisation, the largest size of a cell in the vertical Z-direction $\Delta z$ is $0.04 \mathrm{~m}(H / 3)$ for the coarsest mesh. Thereafter, the mesh is locally refined in all directions in a zone where the free water surface will be located, resulting in $\Delta z=0.02 \mathrm{~m}(H / 6)$. Next, the zone close to the monopile is refined, but only in the vertical direction, leading to $\Delta z=0.01 \mathrm{~m}(H / 12)$. The sizes of each cell in $X$ - and $Y$-direction are based on a maximum aspect ratio (i.e. ratio of largest dimension of a cell over the smallest dimension) of about 2.5. The final grid is presented in Figure 3, which is the coarsest grid for all the simulations performed (case 1) and consists of 397484 cells. Additionally, two more simulations are performed: all cells of case 1 are refined in all directions resulting in case 2 and all cells of case 2 are refined again leading to case 3. During such a refinement, one cell is split up in eight equal cells. A summary of the three cases is given in Table 2. The consecutives lines for each case in column two and three give the cell size for the coarsest part, the zone in which the free water surface will be located and the zone around the monopile respectively. 


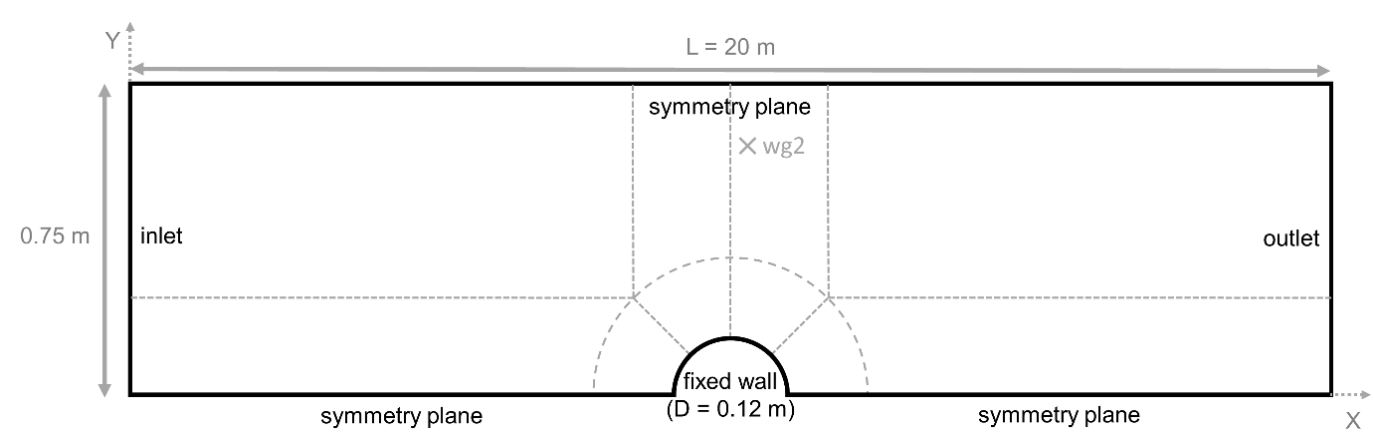

Figure 2: Definition sketch of the geometry of the computational domain in plan view (XY-direction). The dashed lines inside the geometry indicate the internal grid structure whereas the black words characterise the boundary condition type. wg2 ( $x$ $=10.08 \mathrm{~m}, y=0.43 \mathrm{~m}$ ) is a wave gauge used for checking the incoming wave field.

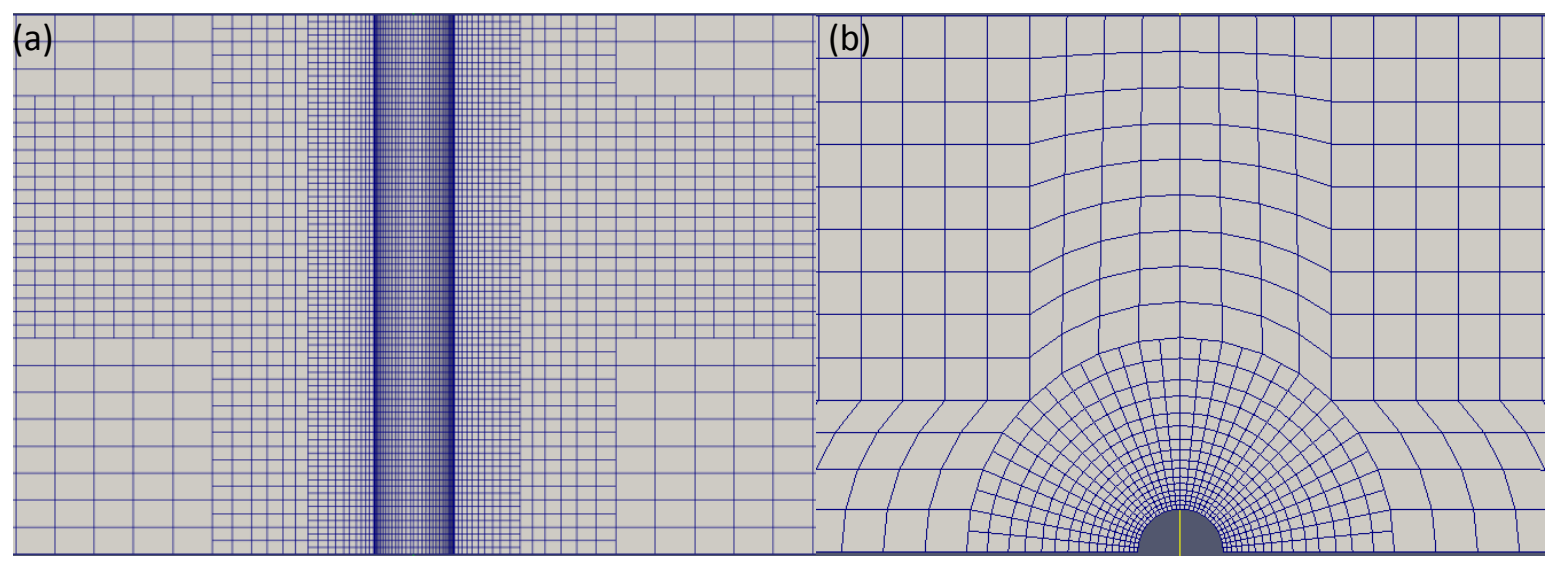

Figure 3: (a) Detail of the longitudinal cross section parallel to the $X Z$-plane through the centre of the monopile. (b) Detailed plan view ( $X Y$-direction) around the monopile.

Table 2: Grid characteristics of the three different cases used. For each case, the first line indicates the overall discretisation. The second line shows the additional refinement in a zone where the free water surface will be located. Finally, the third line shows the final discretisation around the monopile.

\begin{tabular}{c|c|c|c}
\hline case & $\max (\Delta x)$ and $\max (\Delta y)$ & $\Delta z$ & cells \\
\hline \multirow{3}{*}{1} & $0.10 \mathrm{~m}$ & $0.04 \mathrm{~m}(H / 3)$ & \\
& $0.05 \mathrm{~m}$ & $0.02 \mathrm{~m}(H / 6)$ & 397484 \\
& $0.05 \mathrm{~m}$ & $0.01 \mathrm{~m}(H / 12)$ & \\
\hline \multirow{3}{*}{2} & $0.05 \mathrm{~m}$ & $0.02 \mathrm{~m}(H / 6)$ & \\
& $0.025 \mathrm{~m}$ & $0.01 \mathrm{~m}(H / 12)$ & 3179872 \\
& $0.025 \mathrm{~m}$ & $0.005 \mathrm{~m}(H / 24)$ & \\
\hline \multirow{3}{*}{3} & $0.025 \mathrm{~m}$ & $0.01 \mathrm{~m}(H / 12)$ & \\
& $0.0125 \mathrm{~m}$ & $0.005 \mathrm{~m}(H / 24)$ & 25418496 \\
& $0.0125 \mathrm{~m}$ & $0.0025 \mathrm{~m}(H / 48)$ & \\
\hline
\end{tabular}

\subsection{Boundary conditions}

The types of boundary conditions in a horizontal plane are given in Figure 2: inlet on the left, outlet on the right, symmetry plane for the side wall and fixed wall for the monopile. The top of the computational domain is represented by an atmospheric condition while the bottom is a fixed wall.

Two solid walls are modelled, the bottom and the monopile's surface. On both walls, wall functions are activated for $k$ and $\omega$ according to the $k-\omega S S T$ turbulence model. A continuous wall function based on Spalding's law (Spalding, 1961) switching between low- and high-Reynolds numbers is implemented 
for the turbulent viscosity. By using this particular scalable wall function, the dimensionless wall distance $y^{+}$should be between 1 and 300 . On the walls, a Dirichlet boundary condition is set for the velocity ( $0 \mathrm{~m} / \mathrm{s}$ in the three directions) while the pressure and volume fraction are set to a Neumann condition. The atmospheric conditions at the top of the numerical domain are set to a mixed DirichletNeumann boundary condition for the velocity, pressure and volume fraction. The side wall of the wave flume is implemented as a symmetry plane and not as a physical wall to avoid the use of wall functions. This approximation is justified because the ratio of the flume width to the pile diameter $(W / D)$ is 12.5 , which is larger than 6 to avoid side-effects of that wall (Whitehouse, 1998).

At the inlet and outlet, special boundary conditions are needed to generate and absorb waves. Therefore, IHFOAM (Higuera et al., 2013a, 2013b) is deployed as an external toolbox for those boundary conditions. At the inlet, wave generation together with active wave absorption is activated whereas only wave absorption is implemented at the outlet. The implemented wave absorption in IHFOAM is developed by assuming shallow water conditions. However, the waves studied in the present work are close to deep water waves. As mentioned in Higuera et al. (2013a), the absorption function works relatively well outside its range of applicability. Moreover, the reflection coefficient is not below $10 \%$ in experimental laboratories as reported by Cruz (2008). Therefore, it is difficult to compare results when the reflection coefficient is not equal between experimental and numerical setups. The difference between experimental and numerical wave reflection has to be taken into account during the discussion of the results.

During the regular wave tests in the laboratory, wave heights between $0.01 \mathrm{~m}$ and $0.26 \mathrm{~m}$ were generated, with a wave period between $0.40 \mathrm{~s}$ and $2.78 \mathrm{~s}$ resulting in a minimum and maximum wave steepness $s$ of 0.03 and 0.07 respectively. Two regular wave trains with a wave steepness of 0.04 and 0.07 are selected to perform numerical simulations. The wave parameters consist of a fixed wave height $H$ equal to $0.12 \mathrm{~m}$ and a wave period $T$ equal to $1.60 \mathrm{~s}$ and $1.05 \mathrm{~s}$ for wave train 1 and 2 respectively. The water depth $d$ is fixed to $0.50 \mathrm{~m}$. According to Le Méhauté (1969), a $5^{\text {th }}$ order Stokes theory is sufficiently accurate for all the test cases presented.

\subsection{Solver settings}

For all the simulations presented, the following solver settings are used: central discretisation for the pressure gradient and the diffusion terms; TVD (total variation diminishing) schemes with a van Leer limiter (van Leer, 1974) for the divergence operators; backward Euler time discretisation; a maximum Courant number of 0.75 .

\section{Results empty wave flume}

Preliminary simulations are performed in an empty wave flume (i.e. without monopile) in order to assess the quality of the undisturbed propagating wave field over the length of the flume. Therefore, regular waves are generated at the inlet featuring the same wave height and period as the waves used for the study of wave run-up around a monopile.

The two-dimensional (XZ-plane) computational domain is discretised according to the values presented in Table 2. Only the vertical refinement around the monopile is excluded for the empty wave flume simulations (third line for every case). The total number of cells is equal to 9400,37600 and 150400 for case 1 , case 2 and case 3 respectively. For each case, three simulations are performed using the original turbulence model, the buoyancy-modified turbulence model and a laminar model applying the same numerical settings. The laminar result is used as a reference solution because no turbulence is assumed during wave propagation. Moreover, the laminar result will show that numerical 
damping based on RANS turbulence modelling is dominating over possible damping due to the VoF method applied or integration of the RANS equations.

In the following two subsections, time series are provided at particular locations along the flume: $x=$ $1 \mathrm{~m}, x=3 \mathrm{~m}, x=5 \mathrm{~m}, x=10 \mathrm{~m}, x=15 \mathrm{~m}, x=17 \mathrm{~m}$ and $x=19 \mathrm{~m}$. The vertical axis denotes the surface elevation $\eta$ with respect to the bottom $(z=0 \mathrm{~m})$ and the horizontal axis denotes the time for three wave periods after the warming-up phase. Additionally, the turbulent viscosity $v_{t}$ is visualised for both the original and buoyancy-modified turbulence model. Only the results of case 2 (see Table 2) are presented since they are already grid independent.

\subsection{Wave train 1}

This first subsection presents the results of the empty wave flume using the first regular wave train $(H=0.12 \mathrm{~m}, T=1.60 \mathrm{~s}, d=0.50 \mathrm{~m})$. The wave steepness $\mathrm{s}$ is 0.04 and those waves are classified as rather low steepness waves. Figure 4 depicts surface elevations in the empty wave flume for a laminar solution, the original and buoyancy-modified turbulence model. All three simulations return the same time signal at the start of the flume $(x=1 \mathrm{~m})$. Moving towards the end of the flume, small discrepancies in the surface elevation are visible between the original turbulence model and both the laminar model and buoyancy-modified turbulence model. Only a phase shift is observed but the wave height remains constant. As a conclusion, Figure 4 clearly indicates that there is no significant wave damping over the length of the flume based on RANS turbulence modelling for the case of low steepness waves.

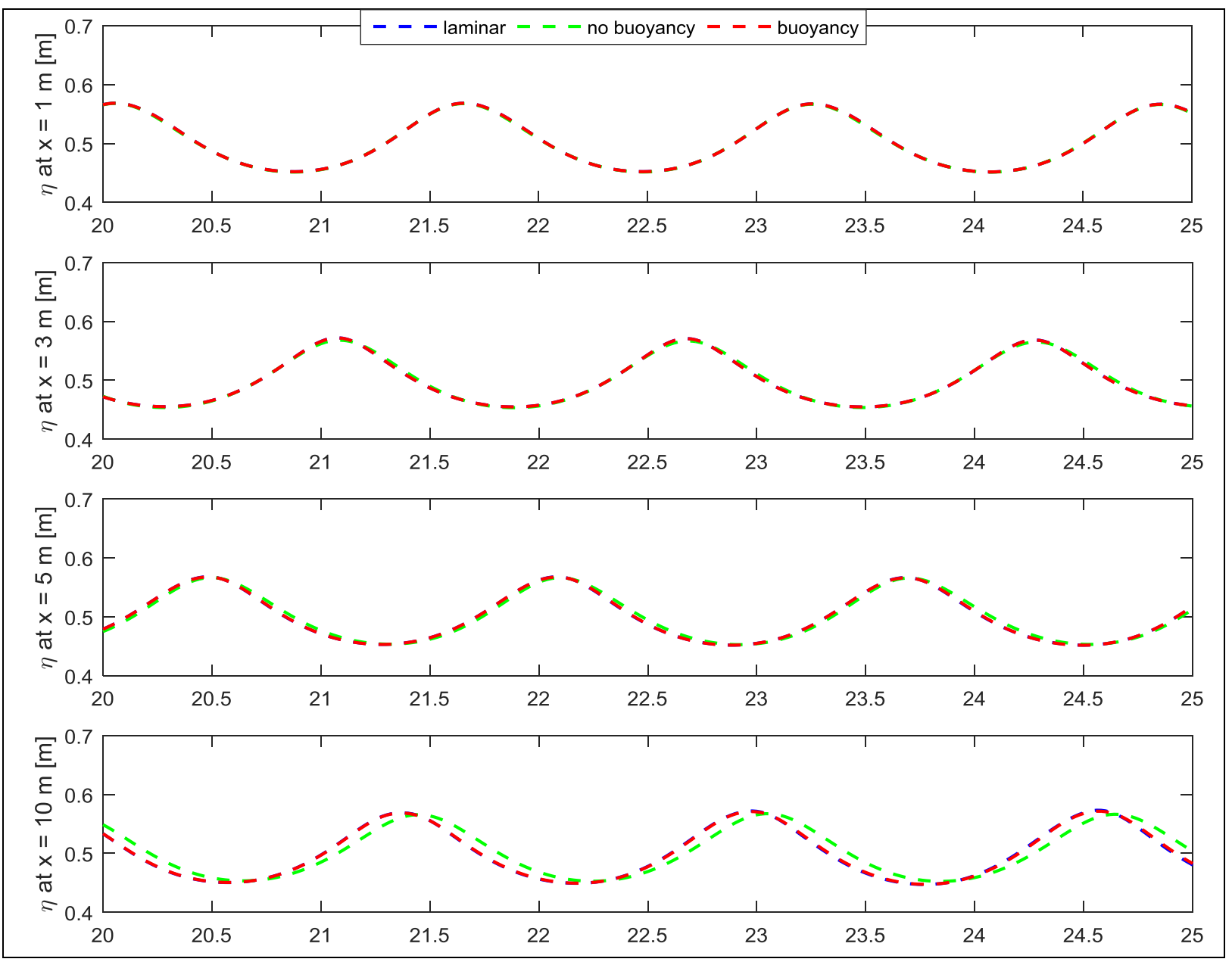




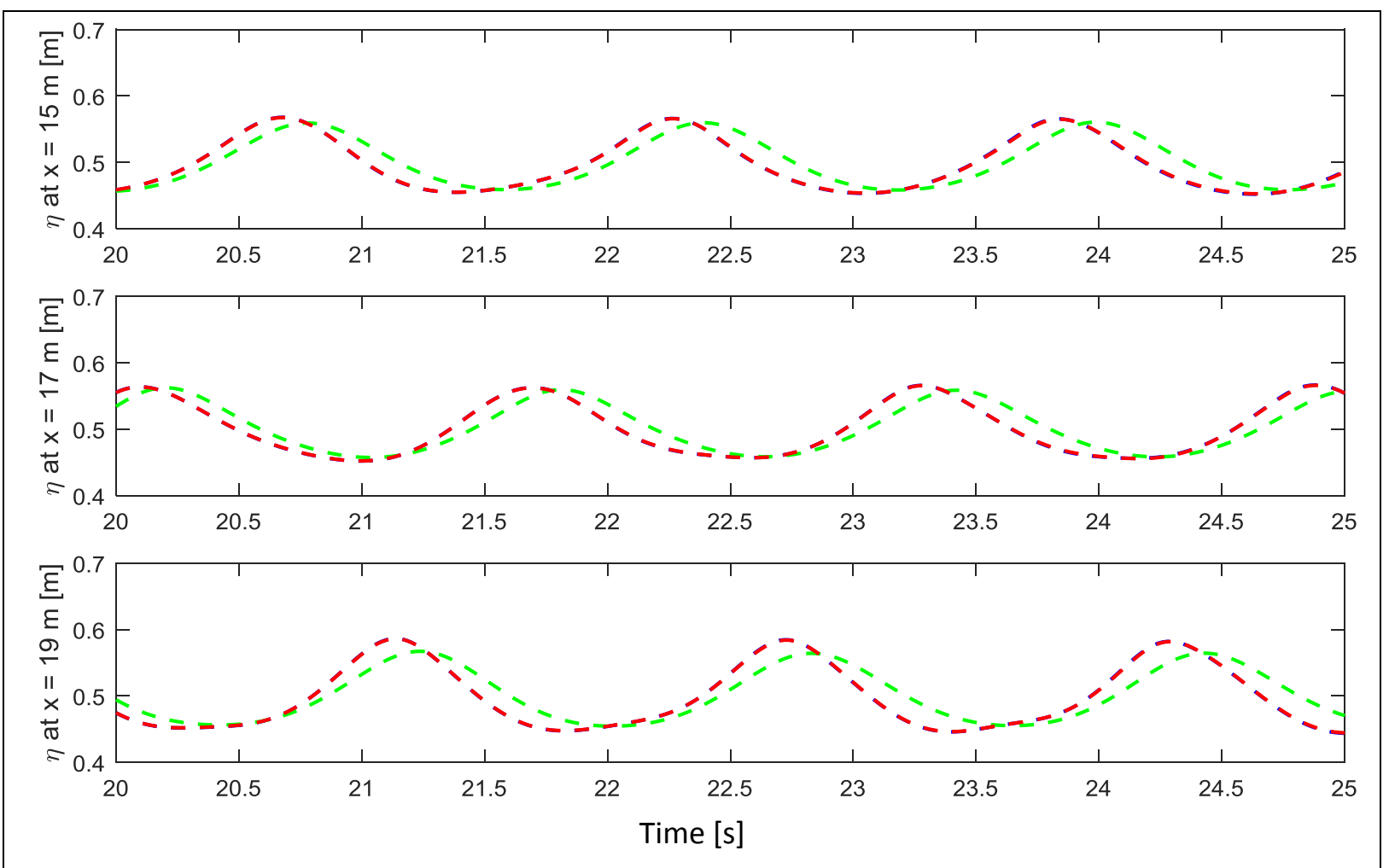

Figure 4: Time series of the surface elevation $\eta$ with respect to the bottom $(z=0 \mathrm{~m})$ from the start $(x=1 \mathrm{~m})$ towards the end $(x=19 \mathrm{~m})$ of the empty wave flume (case 2$)$ for a laminar solution and solutions without and with buoyancy using regular waves: $H=0.12 \mathrm{~m}, T=1.60 \mathrm{~s}, d=0.50 \mathrm{~m}$.

The turbulent viscosity $v_{t}$ for one wave length at the start of the flume is depicted in Figure 5 for both the original and buoyancy-modified turbulence model respectively. The white solid line visualises the location of the free water surface. It is clearly observed that the turbulent viscosity around the free water surface is a few orders of magnitudes larger for the original turbulence model compared to the buoyancy-modified one. However in the case of low steepness waves, this increased viscosity at the free water surface is not affecting the wave height along the length of the wave flume (see Figure 4). 


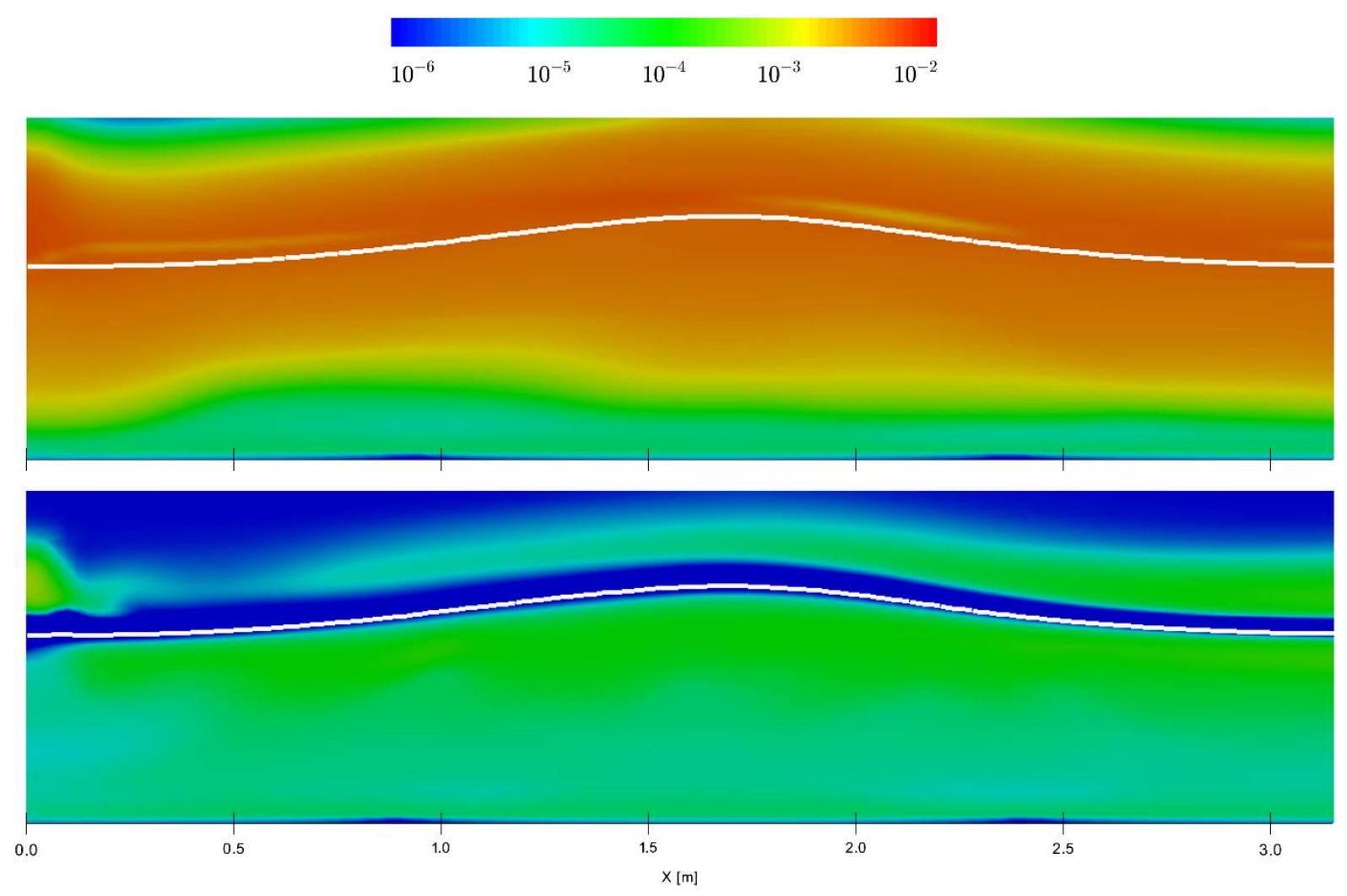

Figure 5: Contour plot of the turbulent kinematic viscosity $v_{t}\left[\mathrm{~m}^{2} / \mathrm{s}\right]$ for one wave length (low steepness waves) at the start of the wave flume $(x=0 \mathrm{~m})$ using a logarithmic scale $(t=22 \mathrm{~s})$ : buoyancy term excluded (top panel) and buoyancy included in the TKE-equation (bottom panel). The white solid line indicates the free water surface.

\subsection{Wave train 2}

This second subsection gives the results of the empty wave flume for the second regular wave train $(H=0.12 \mathrm{~m}, T=1.05 \mathrm{~s}, d=0.50 \mathrm{~m})$. The wave steepness $s$ is now equal to 0.07 and those waves are classified as high steepness waves. Figure 6 depicts surface elevations in the empty wave flume for a laminar solution, the original and buoyancy-modified turbulence model. Close to the wave generation boundary $(x=1 \mathrm{~m})$, the time signal is identical for all the simulations. However, along the length of the flume, a significant decrease of the wave height is observed in case buoyancy is excluded. This damping is not caused by the VoF method since the laminar solution does not show any wave damping. When the buoyancy term is activated, the amplitudes of the surface elevation are close to the laminar solution and do not show any decrease in wave height along the flume. As a conclusion, Figure 6 strongly proves that including a buoyancy term is necessary in order to simulate properly propagating high steepness water waves.

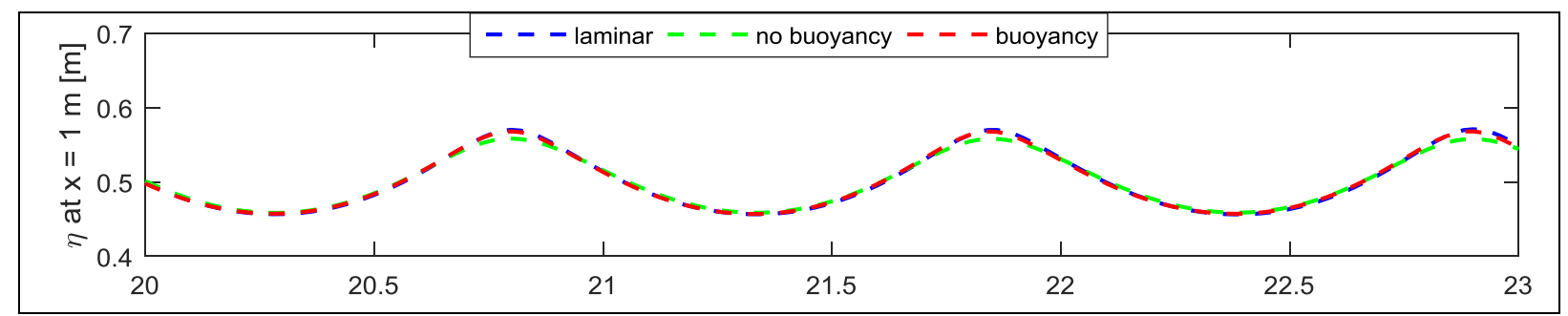



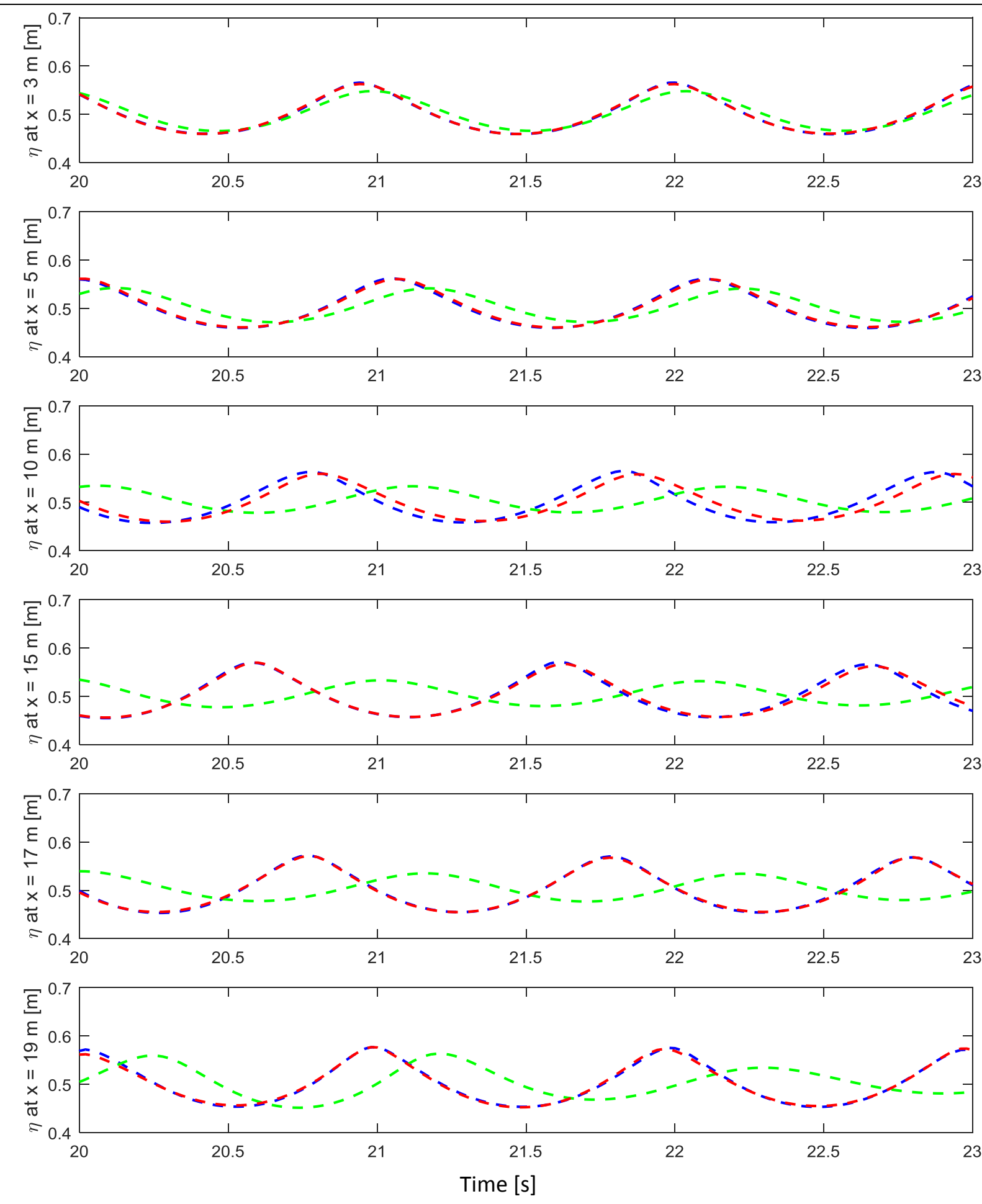

Figure 6: Time series of the surface elevation $\eta$ with respect to the bottom $(z=0 \mathrm{~m})$ from the start $(x=1 \mathrm{~m})$ towards the end $(x=19 \mathrm{~m})$ of the empty wave flume (case 2$)$ for a laminar solution and solutions without and with buoyancy using regular waves: $H=0.12 \mathrm{~m}, T=1.05 \mathrm{~s}, d=0.50 \mathrm{~m}$.

The turbulent viscosity $v_{t}$ for one wave length at the start of the flume is shown in Figure 7 for both the original and buoyancy-modified turbulence model respectively. It is clearly observed that the turbulent viscosity around the free water surface (white solid line) is a few orders of magnitudes larger for the original turbulence model compared to the buoyancy-modified one. As a result for the high steepness waves, the increased viscosity at the free water surface has a significant influence on the wave height along the wave flume (see Figure 6). This increase in turbulent viscosity is the main reason 
for the observed wave damping. However, this wave damping is not observed in case the buoyancy term is included. As mentioned before, the buoyancy term drives the turbulent viscosity around the free water surface to zero and the model switches locally to the laminar regime, preventing excessive wave damping.

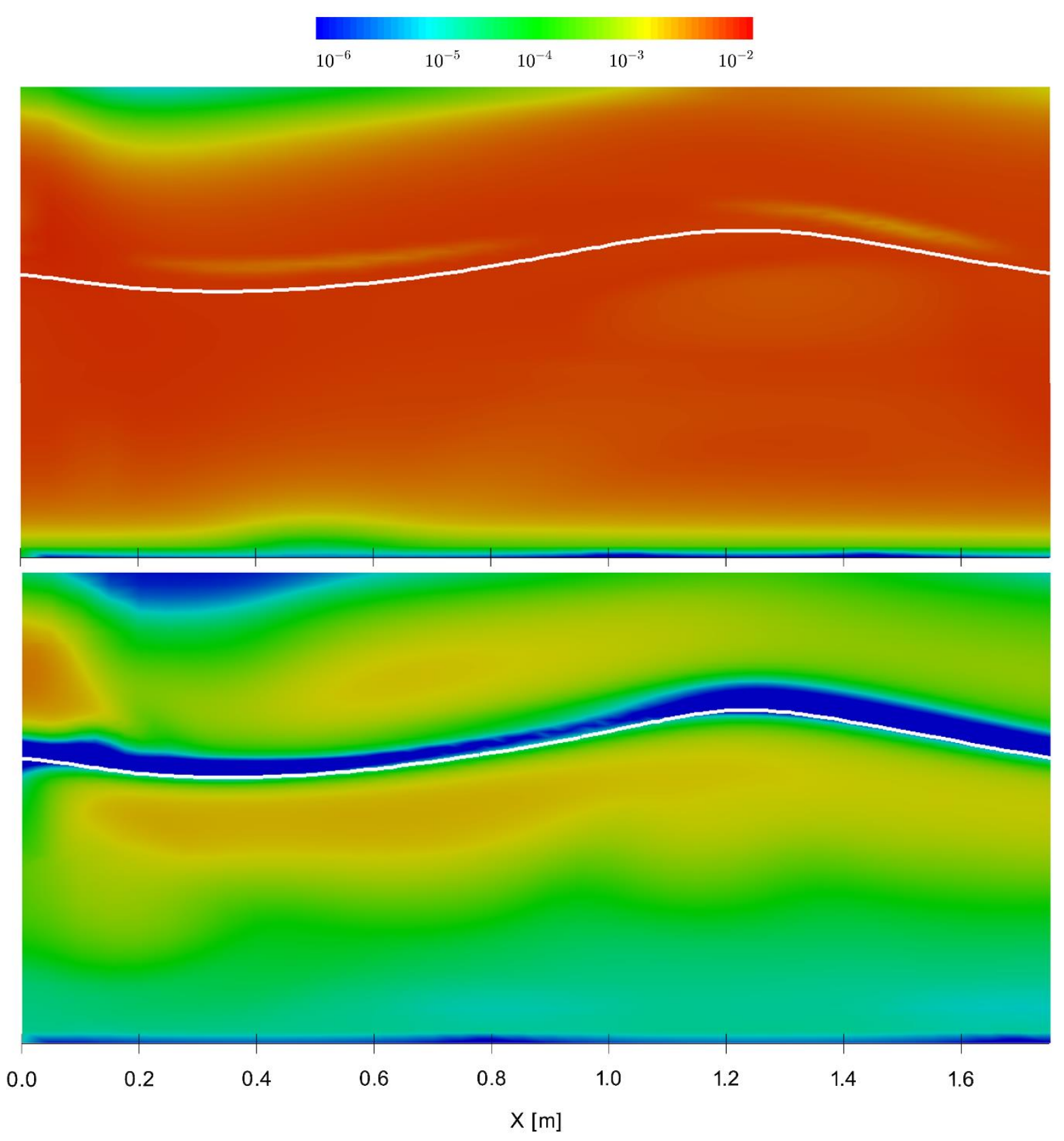

Figure 7: Contour plot of the turbulent kinematic viscosity $v_{t}\left[\mathrm{~m}^{2} / \mathrm{s}\right]$ for one wave length (high steepness waves) at the start of the wave flume $(x=0 \mathrm{~m})$ using a logarithmic scale $(t=22 \mathrm{~s})$ : buoyancy term excluded (top panel) and buoyancy included in the TKE-equation (bottom panel). The white solid line indicates the free water surface.

\section{Results wave run-up around a monopile}

In this section, the numerical obtained wave run-up around a monopile is validated by using laboratory measurements from De Vos et al. (2007). Thereafter, a grid refinement study using the three cases as outlined in Table 2 is reported in order to verify the numerical results. Additionally, numerical results are tested against analytical formulations. Each numerical simulation ran for 20 seconds after the warming-up phase. For every simulation, the quality of the incoming wave field is checked against the 
experimental data at the location of wg2, see Figure 2. As a result, a very similar incoming wave field is observed between the numerical and experimental data.

In the following two subsections, time series of the wave run-up pattern around a monopile are provided in which the vertical axis denotes the surface elevation $\eta$ with respect to the bottom $(z=0$ $\mathrm{m})$. In all the graphs, the horizontal axis denotes the time for three wave periods.

\subsection{Wave train 1}

This first subsection presents the results of wave run-up around a monopile using the first regular wave train $(H=0.12 \mathrm{~m}, T=1.60 \mathrm{~s}, d=0.50 \mathrm{~m})$. As reported in section 4.1, no significant wave damping over the length of the flume based on RANS turbulence modelling for the case of low steepness waves ( $s=$ 0.04 ) is expected. Therefore, the wave run-up pattern around a monopile is presented in the following two paragraphs for respectively excluding and including the buoyancy term.

\subsubsection{Buoyancy term not included in the TKE-equation}

The set of nine graphs displayed in Figure 8 shows the experimental and numerical wave run-up pattern around the monopile without including the buoyancy term in the TKE-equation. The maximum achieved $y^{+}$on the monopile varies from 170 (case 1), 103 (case 2) down to 65 (case 3). Firstly, in general, the same trends in wave run-up are observed for the numerical and experimental data. However, the experimental signal at $112.5^{\circ}$ is not reliable while the numerical model at that location returns plausible results. No reason is found or reported by De Vos et al. (2007) regarding this specific discrepancy. Moreover, Figure 8 clearly indicates that both the shape of the time signal and the local secondary peak in the surface elevation obtained with the numerical model are comparable to the experimental records. Secondly, the numerical solutions seem to be grid independent because the first two grids, case 1 and case 2, are presenting similar results. Interestingly, the numerical results of case 3, show larger discrepancies in the wave run-up pattern compared to case 1 and case 2 . These differences are more significant in front of the monopile (angle wave gauge $<90^{\circ}$ ). It is also remarkable that the largest differences between cases 1 and 2 and case 3 are situated around the wave crest and not around the wave trough.

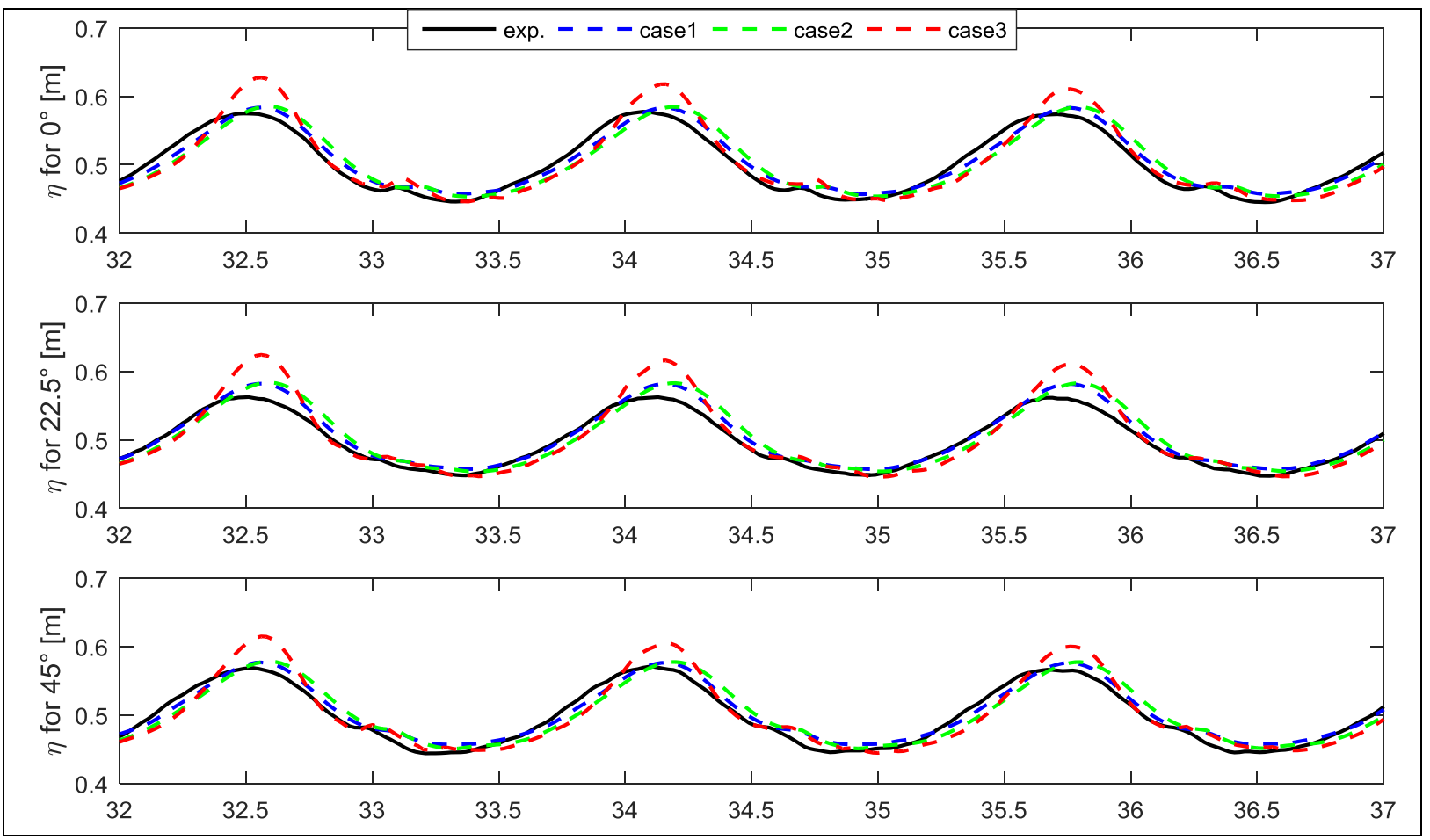



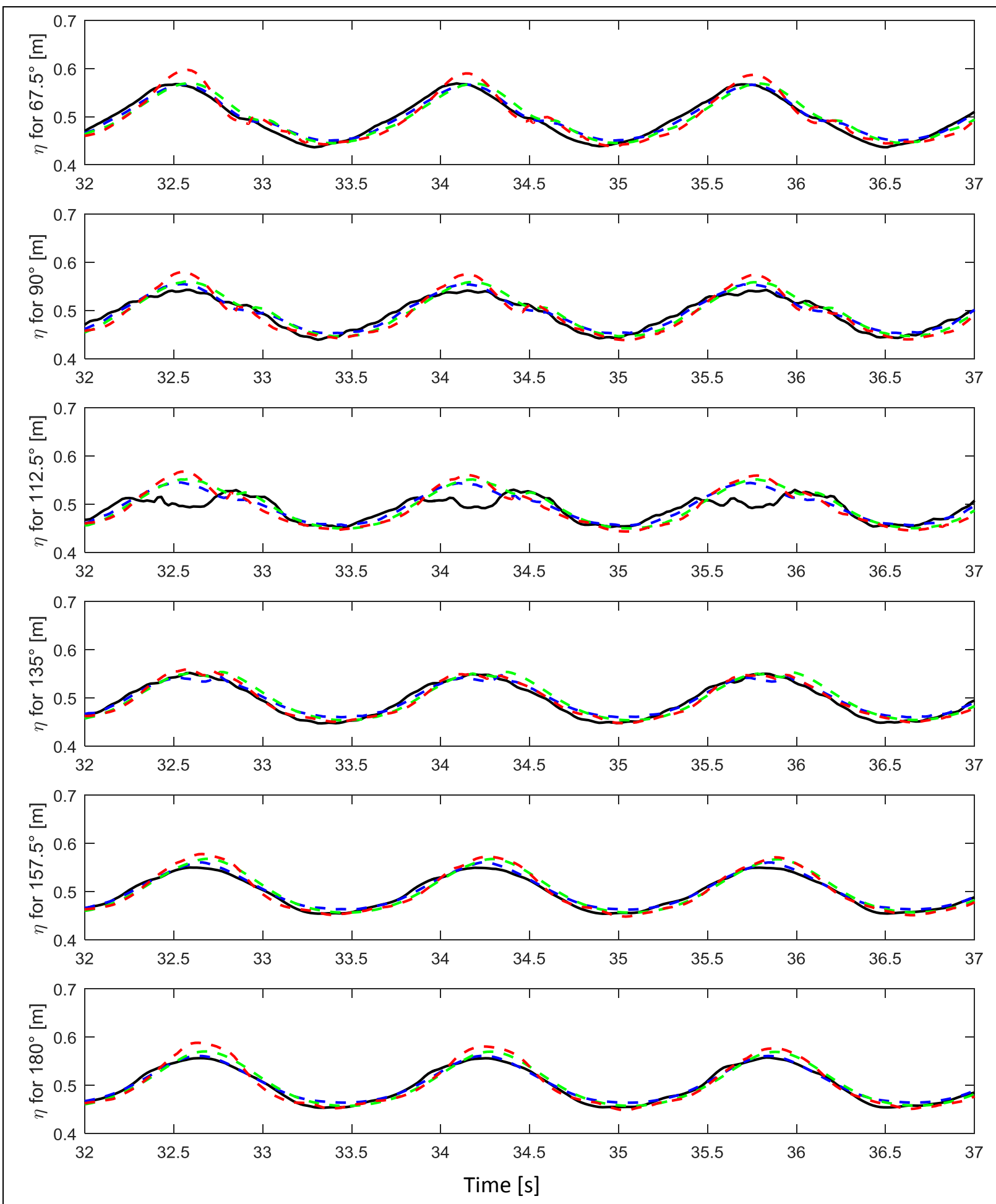

Figure 8: Time series of the wave run-up pattern around the monopile for the experimental data and cases 1, 2 and 3 for different angles of the gauge with respect to the incoming waves. The vertical axis expresses the position of the surface elevation $\eta$ with respect to the bottom $(z=0 \mathrm{~m})$ for regular waves: $H=0.12 \mathrm{~m}, T=1.60 \mathrm{~s}, d=0.50 \mathrm{~m}$, in which the buoyancy term is not included in the TKE-equation.

\subsubsection{Buoyancy term included in the TKE-equation}

In contrast to the previous paragraph, turbulence modelling is now applied by including the buoyancy term in the TKE-equation as formulated in section 3.2. The maximum value of $y^{+}$on the monopile varies from 240 (case 1), 102 (case 2) down to 45 (case 3). Figure 9 presents the wave run-up pattern around the monopile for both the experimental and numerical model. In general, the numerical model 
captures the main effects of wave run-up relatively well. However, the time series indicate clearly that the peak values of the wave run-up are significantly larger in the numerical model compared to the experimental data. Although this difference, the shape, the secondary peak and the surface elevations around the trough are comparable to the experimental time series. Moreover, the solution seems to be grid independent because the three cases are presenting similar results. This means that case 3 is not leading to deflected results as observed in the previous paragraph where buoyancy was not included in the TKE-equation.
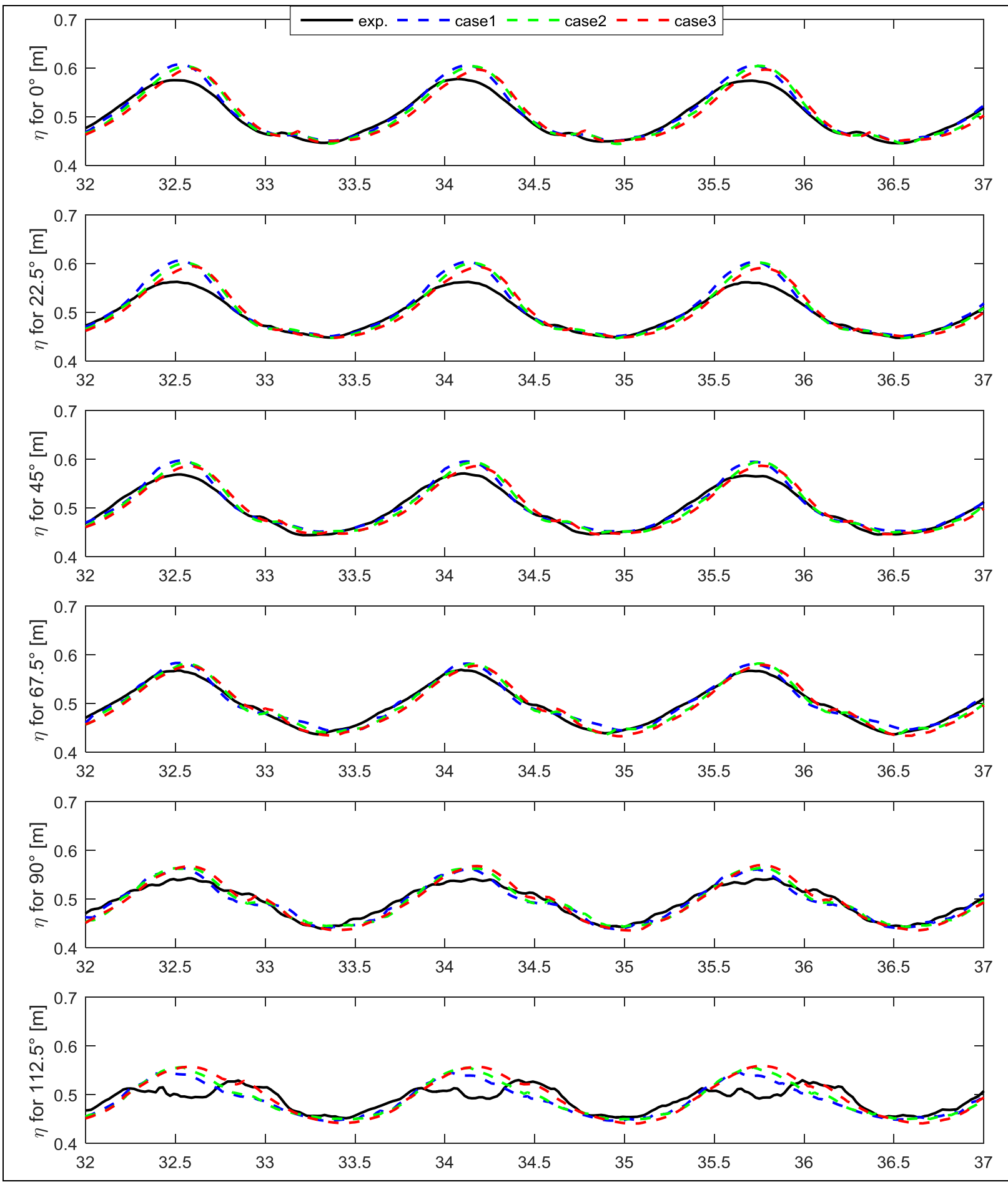


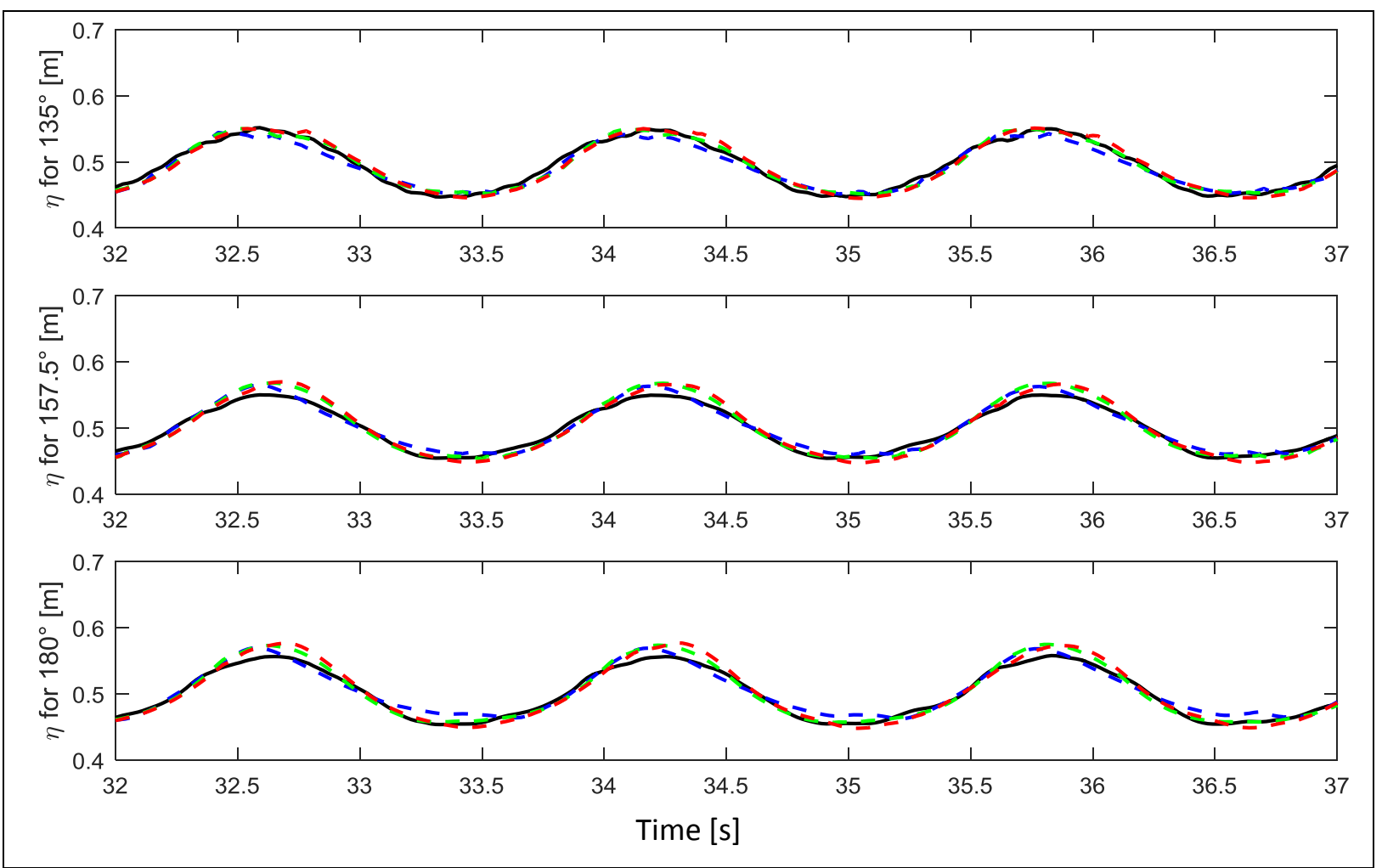

Figure 9: Time series of the wave run-up pattern around the monopile for the experimental data and cases 1, 2 and 3 for different angles of the gauge with respect to the incoming waves. The vertical axis expresses the position of the surface elevation $\eta$ with respect to the bottom $(z=0 \mathrm{~m})$ for regular waves: $H=0.12 \mathrm{~m}, T=1.60 \mathrm{~s}, d=0.50 \mathrm{~m}$, in which the buoyancy term is included in the TKE-equation.

\subsection{Wave train 2}

This second subsection gives the results of wave run-up around a monopile for the second regular wave train ( $H=0.12 \mathrm{~m}, T=1.05 \mathrm{~s}, d=0.50 \mathrm{~m})$. As concluded in section 4.2 , the buoyancy term is needed in the TKE-equation because excessive wave damping over the length of the flume is expected for the case of high steepness waves $(s=0.07)$. Because the incident wave heights are too small close to the monopile in case buoyancy is not activated, only numerical results including buoyancy are presented.

The set of nine graphs given in Figure 10 shows the experimental and numerical wave run-up around the monopile if the buoyancy term is included in the TKE-equation. $y^{+}$on the monopile varies from maximal 300 (case 1), 130 (case 2) down to 70 (case 3). In general, the three numerical cases converge towards a grid independent solution and deliver a comparable output to the experimental results. For example, the local secondary peak in the surface elevation is observed in both numerical and experimental data. However, the peak values of the wave run-up in front of the monopile are sometimes overestimated by the numerical model. Furthermore, the experimental time signal at $90^{\circ}$ is significantly different than the numerically obtained time signals. Several reason can be dedicated for this discrepancy as reported later on in section 6. Moreover, there are some contradictions between experimental and numerical records around the rear part of the monopile.

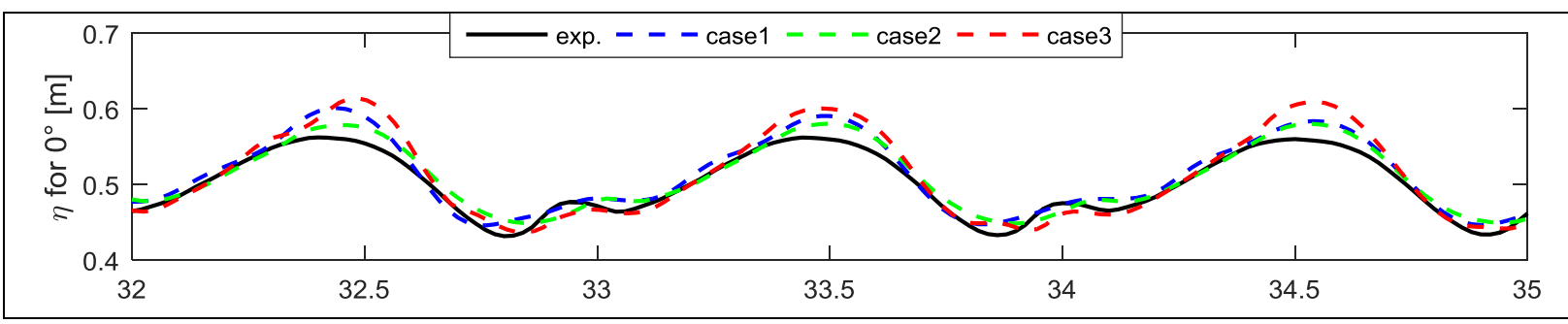



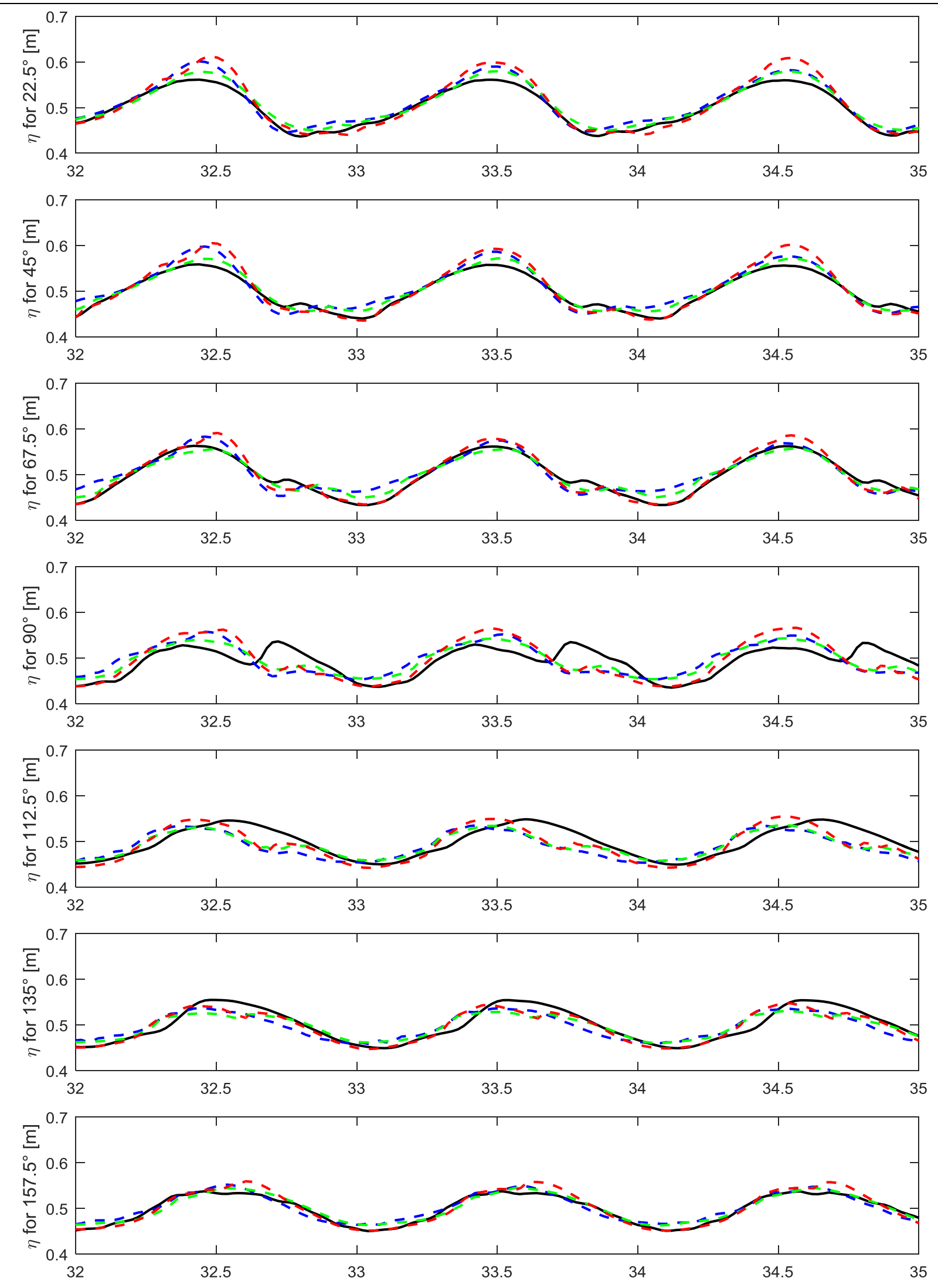


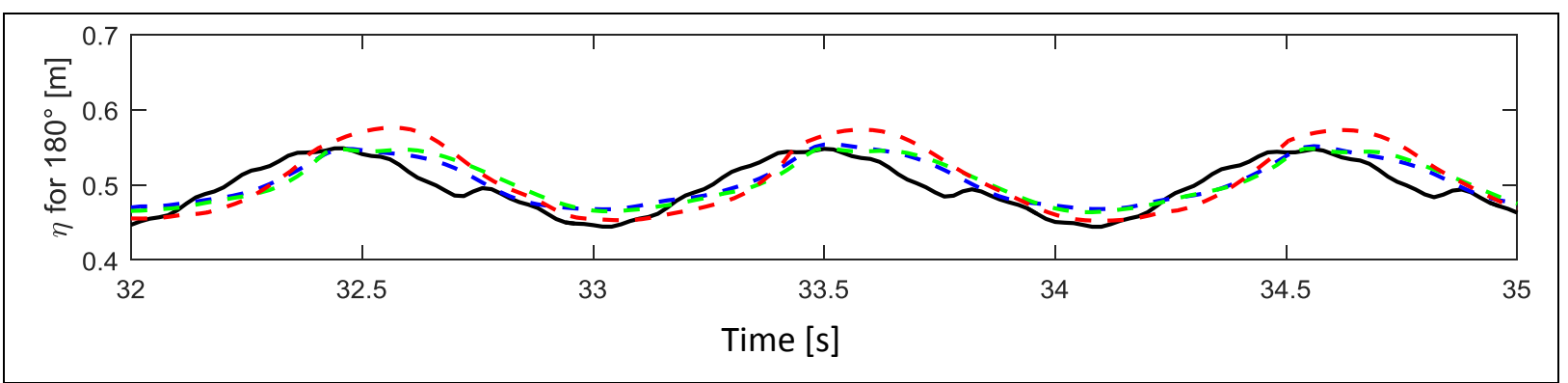

Figure 10: Time series of the wave run-up pattern around the monopile for the experimental data and cases 1,2 and 3 for different angles of the gauge with respect to the incoming waves. The vertical axis expresses the position of the surface elevation $\eta$ with respect to the bottom $(z=0 \mathrm{~m})$ for regular waves: $H=0.12 \mathrm{~m}, T=1.05 \mathrm{~s}, d=0.50 \mathrm{~m}$, in which the buoyancy term is included in the TKE equation.

\subsection{Grid refinement study}

A grid refinement study using the three cases as outlined in Table 2 is performed. A summary is given in Table 3 for the low steepness waves (both original and buoyancy-modified turbulence model) and high steepness waves (buoyancy-modified turbulence model) respectively. The variable used for this analysis is the time-integral of the absolute value of the force on the monopile in $X$-direction over one wave period. This integration is performed when the first wave reaches the outlet boundary. This is needed because wave reflection is strongly dependent on the grid refinement of the mesh (cfr. reflection coefficients reported in section 6$)$. The extrapolated values are computed with Richardson's extrapolation method: $F_{\text {extra. }}=F_{\text {case } 2}+\left(F_{\text {case } 3}-F_{\text {case } 2}\right) /\left(1-2^{-\alpha}\right)$ with $\alpha=\ln \left(\left(F_{\text {case } 1}-F_{\text {case } 2}\right) /\left(F_{\text {case } 2}-\right.\right.$ $\left.\left.F_{\text {case } 3}\right)\right) / \ln (2)$. $F_{\text {extra. }}$ can be regarded as the value calculated from the exact solution. As follows from Table 3, the solutions converge monotonically towards the exact solution.

Table 3: Grid refinement study for the low steepness waves using both the original and buoyancy-modified turbulence model and the high steepness waves using the buoyancy-modified turbulence model. $N_{z}$ is the number of cells in vertical direction next to the cylinder. $F$ is the time-integral of the absolute value of the force on the monopile in $X$-direction over one wave period when the first wave reaches the outlet boundary. $F_{\text {extra. }}$ is the exact solution.

\begin{tabular}{cc|cc|cc|ccc}
\hline \multicolumn{2}{c|}{ Steepness } & \multicolumn{3}{c|}{ Low } & \multicolumn{2}{c}{ High } \\
\hline \multicolumn{2}{c|}{ Buoyancy } & \multicolumn{2}{c|}{ No } & \multicolumn{2}{c}{ Yes } & \multicolumn{2}{c}{ Yes } \\
\hline \multicolumn{2}{c|}{ case } & $N_{z}$ & $F[N s]$ & error & $F[N s]$ & error & $F[N s]$ & error \\
\hline 1 & 80 & 4.921 & $3.59 \%$ & 4.917 & $5.07 \%$ & 4.016 & $9.94 \%$ \\
2 & 160 & 5.048 & $1.10 \%$ & 5.108 & $1.38 \%$ & 4.448 & $0.25 \%$ \\
3 & 320 & 5.087 & $0.34 \%$ & 5.160 & $0.38 \%$ & 4.459 & $0.01 \%$ \\
\hline \multicolumn{2}{c|}{$F_{\text {extra. }}$} & 5.104 & & 5.179 & & 4.459 \\
\hline
\end{tabular}

\subsection{Spatial resolution of wave run-up}

In this subsection, high spatial resolutions of the wave run-up pattern around the monopile over one wave period obtained using the numerical model are presented together with the nine discrete experimental measurements and two analytical formulations. For the experimental dataset, only the maximum wave run-up at the wave gauge's locations over one wave period are shown.

The first analytical expression is reported by De Vos et al. (2007) and is used to calculate the maximum wave run-up around a monopile in case of regular waves:

$$
R_{u}=\eta_{\max }+m \frac{U^{2}}{2 g}
$$


in which $m$ is equal to $1, g$ is the gravitational constant $\left(9.81 \mathrm{~m} / \mathrm{s}^{2}\right), \eta_{\max }$ is the maximum surface elevation and $U$ is the horizontal particle velocity at the wave crest. $\eta_{\max }$ and $U$ are both based on the $2^{\text {nd }}$ order Stokes wave theory. Secondly, Kazeminezhad and Etemad-Shahidi (2015) have recently reanalysed a few experimental datasets and came up with an alternative formulation for the maximum wave run-up level in case of regular waves:

$$
\begin{aligned}
R_{u} & =H\left(0.76\left(\frac{H}{d}\right)^{0.15}\left(\frac{H}{L_{0}}\right)^{-0.055}\right) \quad \text { for } \frac{H}{d} \leq 0.41 \\
R_{u} & =H\left(0.65\left(\frac{H}{L_{0}}\right)^{-0.055}\right) \\
& \left.+3.2 \cdot 10^{-3}\left(\frac{H}{d}-0.41\right)^{0.15}\left(\frac{H}{L_{0}}\right)^{-1.5}\right) \text { for } \frac{H}{d}>0.41
\end{aligned}
$$

in which $H$ is the wave height, $d$ is the water depth and $L_{0}$ the deep water wave length.

The two formulations (14) and (15) lead respectively to a wave run-up level of $0.08 \mathrm{~m}$ and $0.09 \mathrm{~m}$ with respect to the still water level or an absolute value of $0.58 \mathrm{~m}$ and 0.59 with respect to the bottom $(z=0 \mathrm{~m})$ for the first regular wave train $(H=0.12 \mathrm{~m}, T=1.60 \mathrm{~s}, d=0.50 \mathrm{~m})$. Figure 11 shows the wave run-up pattern around the monopile using the numerical model (case 2 ), the experimental obtained wave run-up (dots) and two analytical solutions (horizontal lines). In Figure 11a, the buoyancy term is not included whereas in Figure $11 \mathrm{~b}$ that term is implemented in the TKE-equation. The output of the numerical model is represented by a series of grey lines, each characterising a specific point in time. The time step between two consecutive lines is $0.02 \mathrm{~s}$. As Figure 11 a suggests, the two analytical results are in line with the maximum surface elevation in front of the pile if the buoyancy term is excluded in the numerical model. Moreover, the numerical model shows a significant decrease in the peak value of the wave run-up around $135^{\circ}$. Including buoyancy (Figure $11 \mathrm{~b}$ ) shows that the obtained wave runup is in general higher but it is considerable higher in front of the monopile.

(a)

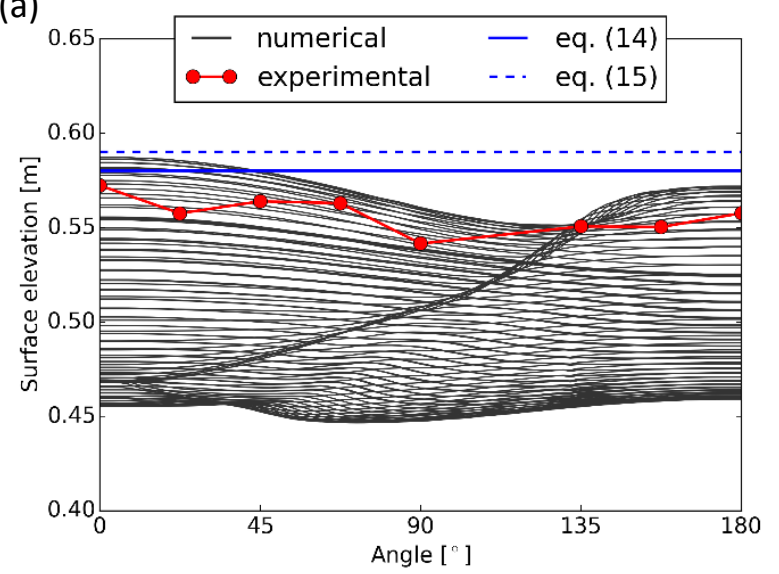

(b)

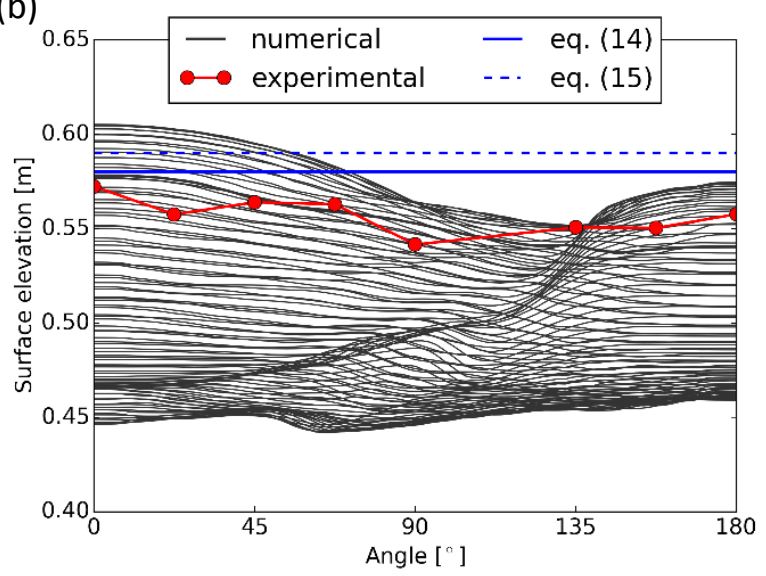

Figure 11: Spatial variance of the wave run-up around the monopile for $y>0$ over one wave period in function of the angle with respect to the incoming waves (wave train 1, case 2): (a) buoyancy term not included and (b) buoyancy term included in the TKE-equation.

In case of the second regular wave train $(H=0.12 \mathrm{~m}, T=1.05 \mathrm{~s}, d=0.50 \mathrm{~m})$, the run-up levels are $0.08 \mathrm{~m}$ and $0.09 \mathrm{~m}$ for equation (14) and (15) respectively. This results in absolute values with respect to the bottom $(z=0 \mathrm{~m})$ of $0.58 \mathrm{~m}$ and $0.59 \mathrm{~m}$ respectively. Figure 12 shows the wave run-up around the monopile for the numerical model (case 2), experimental tests (dots) and two analytical formulae (horizontal lines). Again, each grey line represents numerical data at a certain point in time every $0.02 \mathrm{~s}$. Numerical results are obtained by excluding (Figure 12a) and including the buoyancy term in the TKE-equation (Figure 12b). If the buoyancy term is excluded (Figure 12a), wave run-up around the 
monopile is significant smaller than the experimental data and analytical solutions. This phenomenon is addressed to excessive wave damping over the length of the wave flume, as already discussed in Section 5.2. However, if the buoyancy term is modelled (Figure 12b), the two analytical solutions are in line with the maximum wave run-up in front of the pile. Again, the numerical model shows a significant decrease in the peak value of the wave run-up around $135^{\circ}$.

(a)

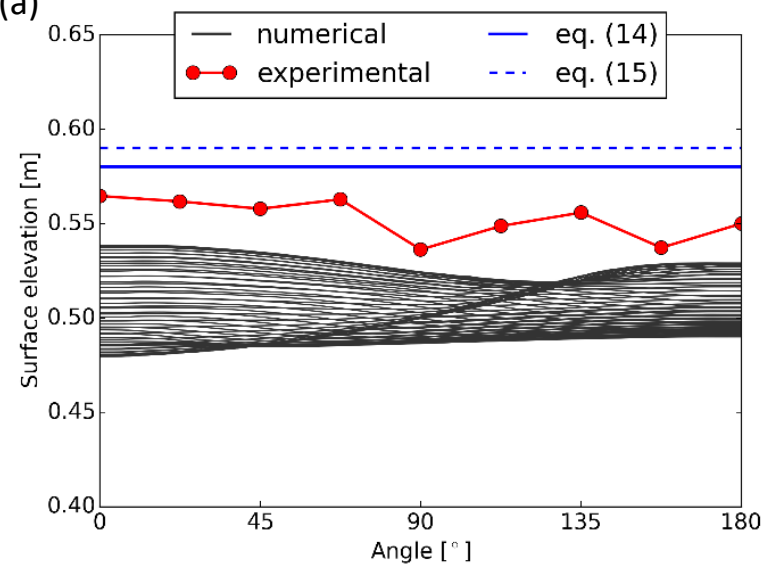

(b)

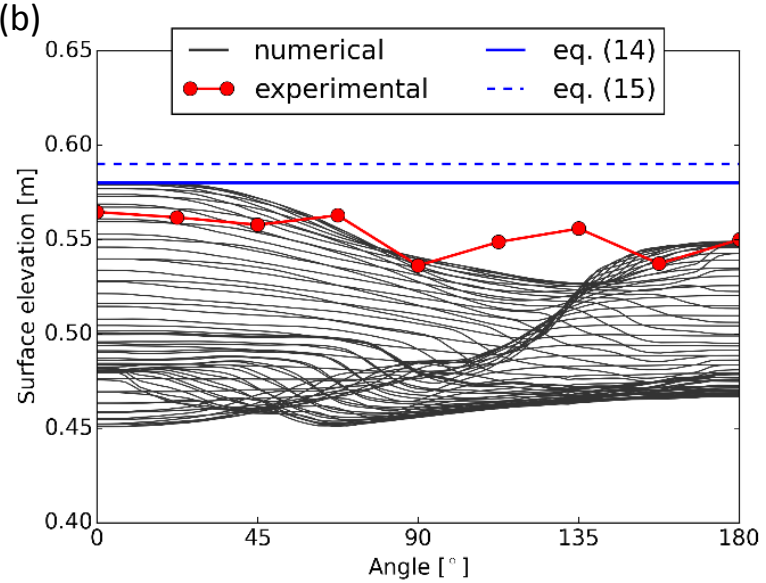

Figure 12: Spatial variance of the wave run-up around the monopile for $y>0$ over one wave period in function of the angle with respect to the incoming waves (wave train 2, case 2): (a) buoyancy term not included and (b) buoyancy term included in the TKE-equation.

\section{Discussion}

Two types of differences in the results are observed. The first one is the difference in numerical results by excluding or including the buoyancy term. For the case of low steepness waves, it is shown that the buoyancy term has only a small effect on the surface elevation over the length of the wave flume. However, there are some larger differences in wave run-up around the monopile between excluding or including the buoyancy term in the TKE-equation. For example, the surface elevations are larger in front of the monopile if buoyancy is taken into account. This observation is related to slightly less damping of the incident waves close to the monopile if buoyancy is modelled. For the case of high steepness waves, the buoyancy term is needed in order to avoid non-physical wave damping over the length of the flume.

The second type of differences is related to the deviations in surface elevations around the monopile between numerical and experimental data. Those deviations are largely assigned to the difference in reflection between the laboratory flume (with an absorbing beach) and the numerical wave flume (with a shallow water absorbing boundary condition). However, De Vos et al. (2007) did not report a value for the reflection coefficient of the absorbing beach in the experimental flume. In this study, the numerical reflection coefficient at the outlet is determined using four wave gauges (at positions $x_{1}=15.5 \mathrm{~m} ; x_{2}=15.808 \mathrm{~m} ; x_{3}=16.3 \mathrm{~m} ; x_{4}=16.5 \mathrm{~m}$; all in the centre of the flume) (Lin and Huang, 2004). For the first wave train without modelling the buoyancy term, the obtained reflection coefficients are $6.4 \%, 5.9 \%$ and $16 \%$ for case 1, case 2 and case 3 respectively. In case buoyancy is activated for the first wave train, the reflection coefficients increase to $15 \%, 13 \%$ and $11 \%$ for case 1 , case 2 and case 3 respectively. These latter values are higher, compared to those where buoyancy is neglected. By including buoyancy in the TKE-equation, less wave damping is observed along the flume, resulting in slightly higher waves reaching the outlet boundary. Because the absorbing correction velocity is directly related to the measured surface elevation at the outlet (Higuera et al., 2013a), the reflected waves are also larger. For the high steepness waves, the obtained reflection coefficients are $23 \%, 22 \%$ and $26 \%$ for case 1 , case 2 and case 3 respectively (with buoyancy modelled). The reflection 
coefficients of this second wave train are significantly higher compared to the first wave train. These high steepness waves are even further away from the shallow water region compared to the low steepness waves, resulting in an inferior performance of the shallow water absorbing boundary condition at the outlet.

In addition, there is also reflection at the inlet boundary of the computational domain from both the reflected waves from the monopile and the outlet. Therefore, active wave absorption (also based on shallow water theory) is activated at the inlet to prevent this re-reflection. Hence, the front part of the monopile is more susceptible for this type of reflected waves. However, no qualitative method is used to assess this re-reflection from the inlet boundary.

A minor source of deviations between numerical and experimental results is the measurement technique for the wave run-up level. However, De Vos et al. (2007) reported no details about the accuracy of the experimental wave run-up measurements. The experimental measurements may be influenced by the presence of the wave gauges (two cylindrical rods) near the pile, while in the numerical model the wave gauges are not modelled (wave run-up is measured th that same location however). This effect is considered marginal in contrast to the difference in reflection between experimental and numerical models.

Despite the observed inaccuracies, in general the numerical results are in good agreement with the experimentally obtained wave run-up levels. It may be expected that the inaccuracies related to the experimental measurements also contribute to the achieved level of agreement. In contrast to the experimental data, which are only available at nine discrete locations, the numerical model yields a much higher spatial resolution of the wave run-up levels around the monopile. As a result, the wave run-up phenomenon features are much easier to identify using the numerical toolbox.

\section{Conclusions and future work}

In this paper, we presented a buoyancy-modified $k-\omega S S T$ turbulence model that prevents an excessive decrease in wave height over the length of the numerical wave flume based on RANS turbulence modelling. Therefore, a buoyancy term has been implemented in the TKE-equation in order to develop an overall stable wave propagation model without significant wave damping over the length of the flume. The influence of that buoyancy term was demonstrated by performing wave propagation simulations in an empty wave flume using $\mathrm{OpenFOAM}^{\circledR}$. Subsequently, we validated this buoyancymodified turbulence model by simulating wave run-up around a monopile subjected to regular waves. Therefore, two regular wave trains were generated, each with a different wave steepness. The obtained numerical results of the wave run-up pattern around the monopile show a fair agreement with experimental data and two analytical formulations. The outcome of this paper proves the successful capability of a buoyancy-modified turbulence model in order to simulate offshore and coastal engineering processes. Future work includes the validation of wave breaking processes using the buoyancy-modified $k-\omega S S T$ turbulence model. The buoyancy term will not only result in a stable wave propagation model without significant wave damping over the length of the flume but it will also predict the turbulence level in the flow field more accurately at the locations where wave breaking occurs.

\section{Acknowledgement}

The first author is Ph.D. fellow of the Research Foundation - Flanders (FWO), Belgium (Ph.D. fellowship $1133815 N)$. Part of the research has been carried out within the FWO research Project 3G029114. Leen De Vos is also gratefully acknowledged to make her experimental data available for this research. 


\section{References}

Brown, S.A., Magar, V., Greaves, D.M., Conley, D.C., 2014. An Evaluation of RANS Turbulence Closure Models for Spilling Breakers. Coast. Eng. Proc. 1, 5. doi:10.1126/science.164.3879.590

Chen, L.F., Zang, J., Hillis, a. J., Morgan, G.C.J., Plummer, a. R., 2014. Numerical investigation of wavestructure interaction using OpenFOAM. Ocean Eng. 88, 91-109. doi:10.1016/j.oceaneng.2014.06.003

Christensen, E.D., Bredmose, H., Hansen, E.A., 2005. Extreme wave forces and wave run-up on offshore wind-turbine foundations. Copenhagen Offshore Wind 2005 Conf. 1-10.

Cruz, J., 2008. Ocean Wave Energy: Current Status and Future Perspectives.

De Vos, L., Frigaard, P., De Rouck, J., 2007. Wave run-up on cylindrical and cone shaped foundations for offshore wind turbines. Coast. Eng. 54, 17-29. doi:10.1016/j.coastaleng.2006.08.004

El Safti, H., Bonakdar, L., Oumeraci, H., 2014. A Hybrid 2D-3D CFD Model System for Offshore Pile Groups Subject to Wave Loading, in: Volume 2: CFD and VIV. ASME, p. V002T08A040. doi:10.1115/OMAE2014-23636

Elhanafi, A., Fleming, A., Leong, Z., MacFarlane, G., n.d. Effect of RANS-based Turbulence Models on Nonlinear Wave Generation in a Two-Phase Numerical Wave Tank. Prog. Comput. Fluid Dyn. 118.

Higuera, P., Lara, J.L., Losada, I.J., 2013a. Realistic wave generation and active wave absorption for Navier-Stokes models. Application to OpenFOAM. Coast. Eng. 71, 102-118. doi:10.1016/j.coastaleng.2012.07.002

Higuera, P., Lara, J.L., Losada, I.J., 2013b. Simulating coastal engineering processes with OpenFOAM. Coast. Eng. 71, 119-134. doi:10.1016/j.coastaleng.2012.06.002

Hirt, C.W., Nichols, B.D., 1981. Volume of Fluid (VoF) Method for the Dynamics of Free Boundaries. J. Comput. Phys. 39, 201-225. doi:10.1016/0021-9991(81)90145-5

Jacobsen, N.G., Fuhrman, D.R., Fredsøe, J., 2012. A wave generation toolbox for the open-source CFD library: OpenFoam ${ }^{\circledR}$. Int. J. Numer. Methods Fluids 70, 1073-1088. doi:10.1002/fld.2726

Kamath, A., Alagan Chella, M., Bihs, H., Arntsen, Øivind A., 2015a. Evaluating wave forces on groups of three and nine cylinders using a 3D numerical wave tank. Eng. Appl. Comput. Fluid Mech. 9, 343-354. doi:10.1080/19942060.2015.1031318

Kamath, A., Chella, M.A., Bihs, H., Arntsen, Ø.A., 2015b. CFD investigations of wave interaction with a pair of large tandem cylinders. Ocean Eng. 108, 738-748. doi:10.1016/j.oceaneng.2015.08.049

Kazeminezhad, M.H., Etemad-Shahidi, A., 2015. A new method for the prediction of wave runup on vertical piles. Coast. Eng. 98, 55-64. doi:10.1016/j.coastaleng.2015.01.004

Lara, J.L., Higuera, P., Guanche, R., Losada, I.J., 2013. Wave Interaction With Piled Structures: Application With IH-FOAM, in: Volume 7: CFD and VIV. ASME, p. V007T08A078. doi:10.1115/OMAE2013-11479

Le Méhauté, B., 1969. An Introduction to Hydrodynamics and Water Waves. Ocean. Front. Coast. Process.

Lin, C.Y., Huang, C.J., 2004. Decomposition of incident and reflected higher harmonic waves using four wave gauges. Coast. Eng. 51, 395-406. doi:10.1016/j.coastaleng.2004.04.004

Mayer, S., Madsen, P.A., 2000. Simulation of Breaking Waves in the Surf Zone using a Navier-Stokes Solver. Coast. Eng. 928-941. doi:http://dx.doi.org/10.1061/40549(276)72 
Menter, F.R., Ferreira, J.C., Esch, T., 2003. The SST Turbulence Model with Improved Wall Treatment for Heat Transfer Predictions in Gas Turbines. Int. Gas Turbine Congr. 2003 1-7.

OpenFOAM $^{\circledR}$, 2013. OpenFOAM-2.2.2. URL http://www.openfoam.org/

Paulsen, B.T., Bredmose, H., Bingham, H.B., 2014. An efficient domain decomposition strategy for wave loads on surface piercing circular cylinders. Coast. Eng. 86, 57-76. doi:10.1016/j.coastaleng.2014.01.006

Peng, Z., Wellens, P., Raaijmakers, T., 2012. 3-D Numerical Modeling of Wave Run-Up on Monopiles, in: Volume 5: Ocean Engineering; CFD and VIV. ASME, p. 327. doi:10.1115/OMAE2012-83858

Rahman, M.M., Karim, M.M., Alim, M.A., 2008. Numerical investigation of unsteady flow past a circular cylinder using 2-D finite volume method. J. Nav. Archit. Mar. Eng. 4, 27-42. doi:10.3329/jname.v4i1.914

Ransley, E., Hann, M., Greaves, D., Raby, A., Simmonds, D., 2013. Numerical and Physical Modelling of Extreme Wave Impacts on a Fixed Truncated Circular Cylinder, in: Ewtec.

Saghafian, M., Stansby, P.K., Saidi, M.S., Apsley, D.D., 2003. Simulation of turbulent flows around a circular cylinder using nonlinear eddy-viscosity modelling: steady and oscillatory ambient flows. J. Fluids Struct. 17, 1213-1236. doi:10.1016/S0889-9746(03)00066-5

Spalding, D.B., 1961. A Single Formula for the "Law of the Wall." J. Appl. Mech. 28, 455. doi:10.1115/1.3641728

Sumer, B., Fredsøe, J., 1997. Hydrodynamics around cylindrical structures.

van Leer, B., 1974. Towards the ultimate conservative difference scheme. II. Monotonicity and conservation combined in a second-order scheme. J. Comput. Phys. 14, 361-370. doi:10.1016/0021-9991(74)90019-9

Van Maele, K., Merci, B., 2006. Application of two buoyancy-modified k- $\varepsilon$ turbulence models to different types of buoyant plumes. Fire Saf. J. 41, 122-138. doi:10.1016/j.firesaf.2005.11.003

Vanneste, D., Troch, P., 2015. 2D numerical simulation of large-scale physical model tests of wave interaction with a rubble-mound breakwater. Coast. Eng. 103, 22-41. doi:10.1016/j.coastaleng.2015.05.008

Whitehouse, R., 1998. Scour at marine structures: A manual for practical applications. 